\title{
Local polar invariants and the Poincaré problem in the dicritical case
}

\author{
By Yohann Genzmer and Rogério MoL
}

(Received Oct. 11, 2016)

(Revised Apr. 1, 2017)

\begin{abstract}
We develop a study on local polar invariants of planar complex analytic foliations at $\left(\mathbb{C}^{2}, 0\right)$, which leads to the characterization of second type foliations and of generalized curve foliations, as well as to a description of the $G S V$-index. We apply it to the Poincaré problem for foliations on the complex projective plane $\mathbb{P}_{\mathbb{C}}^{2}$, establishing, in the dicritical case, conditions for the existence of a bound for the degree of an invariant algebraic curve $S$ in terms of the degree of the foliation $\mathcal{F}$. We characterize the existence of a solution for the Poincaré problem in terms of the structure of the set of local separatrices of $\mathcal{F}$ over the curve $S$. Our method, in particular, recovers the known solution for the non-dicritical case, $\operatorname{deg}(S) \leq \operatorname{deg}(\mathcal{F})+2$.
\end{abstract}

\section{Introduction.}

Let $\mathcal{F}$ be a singular holomorphic foliation on $\mathbb{P}_{\mathbb{C}}^{2}$. The number of points of tangency, with multiplicities counted, between $\mathcal{F}$ and a non-invariant line $L \subset \mathbb{P}_{\mathbb{C}}^{2}$ is the degree of the foliation and is denoted by $\operatorname{deg}(\mathcal{F})$. In [27], Poincaré proposed the problem of bounding the degree of an algebraic curve $S$ invariant by $\mathcal{F}$ in terms of $\operatorname{deg}(\mathcal{F})$ as a step in finding a rational first integral for a polynomial differential equation in two complex variables. Known in Foliation Theory as the Poincaré problem, along the past few decades this problem has gained some partial answers. In 1991, Cerveau and Lins Neto proved in [13] that if $S$ has at most nodal singularities then $\operatorname{deg}(S) \leq \operatorname{deg}(\mathcal{F})+2$, this bound being reached if and only if $\mathcal{F}$ is a logarithmic foliation - one induced by a closed meromorphic 1-form with simple poles. Later, in 1994, Carnicer obtained in [10] the same inequality when the singularities of $\mathcal{F}$ over $S$ are all non-dicritical, meaning that the number of local separatrices - local irreducible invariant curves — is finite. In 1997, in the works $[3]$ and [4], Brunella formulated the Poincaré problem in terms of $G S V$-indices, defined by Gómez-Mont, Seade and Verjovsky in [19] as a kind of Poincaré-Hopf index of the restriction to an invariant curve of a vector field tangent to $\mathcal{F}$. To wit, Brunella shows that the bound $\operatorname{deg}(S) \leq \operatorname{deg}(\mathcal{F})+2$ occurs whenever the sum over $S$ of the $G S V$-indices of $\mathcal{F}$ with respect to the local branches of $S$ is non-negative [4, p.533]. It is well known that, in general, the Poincaré problem has a negative answer in the dicritical case (see

2010 Mathematics Subject Classification. Primary 32S65.

Key Words and Phrases. holomorphic foliation, invariant curves, Poincaré problem, GSV-index.

This work was supported by MATH-AmSud Project CNRS/CAPES/Concytec. The first author was supported by a grant ANR-13-JS01-0002-0. The second author was supported by Pronex/FAPERJ and Universal/CNPq. 
6.1 and 6.2 below). Some advances in the understanding of the dicritical case have been made in the past years, as shown in the works $[\mathbf{7}],[\mathbf{8}],[\mathbf{1 6}],[\mathbf{1 2}],[\mathbf{1 7}]$.

The study of global invariant curves leads us to the universe of local foliations on $\left(\mathbb{C}^{2}, 0\right)$, in which we distinguish two families with relevant properties. First, generalized curve foliations, defined in [5] by Camacho, Lins Neto and Sad, which are foliations without saddle-nodes in their desingularization. They have a property of minimization of Milnor numbers and are characterized, in the non-dicritical case, by the vanishing of the $G S V$-index [4], [11]. The second family, which contains the first one, is formed by second type foliations, introduced by Mattei and Salem in [22], which may admit saddles-nodes when desingularized provided that they are non-tangent saddles-nodes, meaning that no weak separatrix is contained in the desingularization divisor. They are characterized by the fact that their desingularizations coincide with the reduction of the set of formal separatrices. These foliations satisfy a property of minimization of the algebraic multiplicity $[\mathbf{2 2}],[\mathbf{1 8}]$.

In a recent work [9], Cano, Corral and Mol developed a study of local polar invariants obtaining, in the non-dicritical case, a characterization of generalized curves and of foliations of second type as well as an expression of the $G S V$-index in terms of these invariants. Essentially, the technique therein consists in calculating the intersection number between a generic polar curve of a local foliation $\mathcal{F}$ and a curve of formal separatrices $C$. The same number is produced for the formal "reference foliation" having the local equation of $C$ as a first integral. The difference of these two numbers is the $G S V$-index of $\mathcal{F}$ with respect to $C$. In this way, the known answers to the Poincaré problem just mentioned are obtained.

In this paper we extend this approach to dicritical foliations - those with infinitely many separatrices. The difficulty now lies in choosing a finite set of separatrices in order to produce such a "reference foliation". The solution is to use a balanced equation of separatrices, a concept introduced by Genzmer in $[\mathbf{1 8}]$ for the study of the "realization problem" - the existence of foliations with prescribed reduction of singularities and projective holonomy representations. Given a local foliation $\widehat{\mathcal{F}}$ at $\left(\mathbb{C}^{2}, 0\right)$ with minimal reduction of singularities $E:(\mathcal{M}, \mathcal{D}) \rightarrow\left(\mathbb{C}^{2}, 0\right)$, an irreducible component $D \subset \mathcal{D}$ is said to be non-dicritical — respectively dicritical — if it is invariant — respectively noninvariant - by the strict transform foliation $E^{*} \widehat{\mathcal{F}}$. The valence of $D \subset \mathcal{D}$ is the number $\mathrm{v}(D)$ of other components of $\mathcal{D}$ intersecting $D$. A balanced equation of separatrices turns out to be a formal meromorphic function $\hat{F}$ that encompasses the equations of all isolated separatrices - the ones crossing non-dicritical components of $\mathcal{D}$ - along with the equations of $2-\mathrm{v}(D)$ separatrices associated to each dicritical component $D \subset \mathcal{D}$. This can be a negative number, so dicritical separatrices may engender poles in the balanced equation. We can additionally adjust this definition when a local set of separatrices $C$ for $\widehat{\mathcal{F}}$ is fixed in order to get a balanced equation adapted to $C$. This is achieved by rebalancing the number of dicritical separatrices of $\hat{F}$ in such a way that $C \subset(\hat{F})_{0}$. We develop these concepts in Section 3.

In Section 4, our starting point is the extension of the definition of the polar intersection number introduced in [9] to a formal meromorphic 1-form, which will normally be $\mathrm{d} \hat{F}$, where $\hat{F}$ is a balanced equation of separatrices of the foliation $\widehat{\mathcal{F}}$. For a fixed set of separatrices $C$ of $\widehat{\mathcal{F}}$, the comparison of the polar intersection numbers of $\widehat{\mathcal{F}}$ and $\mathrm{d} \hat{F}$, 
where $\hat{F}$ is a balanced equation of separatrices such that $C \subset(\hat{F})_{0}$, gives rise to the polar excess index, denoted by $\Delta_{p}(\widehat{\mathcal{F}}, C)$. This non-negative invariant works as a measure of the existence of saddles-nodes in the desingularization of the foliation: $\Delta_{p}\left(\widehat{\mathcal{F}},(F)_{0}\right)=0$ if and only if $\widehat{\mathcal{F}}$ is a generalized curve. This is the content of Theorem A, which extends to the dicritical case the characterization of generalized curve foliations provided, in the non-dicritical case, by the vanishing of the $G S V$-index with respect to the complete set of separatrices. Actually, in Section 5, Theorem B establishes a link between the polar excess and the $G S V$-index for a convergent set of separatrices:

$$
G S V_{p}(\widehat{\mathcal{F}}, C)=\Delta_{p}(\widehat{\mathcal{F}}, C)+\left(C,(\hat{F})_{0} \backslash C\right)_{p}-\left(C,(\hat{F})_{\infty}\right)_{p}
$$

where $\hat{F}$ is a balanced equation of separatrices such that $C \subset(\hat{F})_{0}$. Here, $\left(S_{1}, S_{2}\right)_{p}$ stands for the intersection number of two germs at $p$ of curves $S_{1}$ and $S_{2}$ defined by

$$
\left(S_{1}, S_{2}\right)_{p}=\operatorname{dim}_{\mathbb{C}} \frac{\mathcal{O}_{p}}{\left(f_{1}, f_{2}\right)},
$$

where $f_{1}$ and $f_{2}$ are reduced local equation of $S_{1}$ and $S_{2}$. Notice that, when $\widehat{\mathcal{F}}$ is nondicritical and $C$ is the complete set of separatrices, this gives $G S V_{p}(\widehat{\mathcal{F}}, C)=\Delta_{p}(\widehat{\mathcal{F}}, C)$.

This formulation of the $G S V$-index enables us in Theorem $\mathrm{C}$ in Section 6 to propose a bound to the Poincaré problem in terms of local balanced sets of separatrices. For a foliation $\mathcal{F}$ on $\mathbb{P}_{\mathbb{C}}^{2}$ having an invariant algebraic curve $S$, if $d=\operatorname{deg}(\mathcal{F})$ and $d_{0}=\operatorname{deg}(S)$, it holds

$$
d_{0} \leq d+2+\frac{1}{d_{0}} \sum_{p \in \operatorname{Sing}(\mathcal{F}) \cap S}\left[\left(S,\left(\hat{F}_{p}\right)_{\infty}\right)_{p}-\left(S,\left(\hat{F}_{p}\right)_{0} \backslash S\right)_{p}\right]
$$

where $\hat{F}_{p}$ is a balanced equation adapted to the local branches of $S$. Besides, equality holds if all singularities of $\mathcal{F}$ over $S$ are generalized curves. In particular, this inequality recovers the bound $d_{0} \leq d+2$ for the cases treated in [13] and [10].

In the right side of the previous formula, the terms $\left(S,\left(\hat{F}_{p}\right)_{\infty}\right)_{p}$ are obstructions to the existence of a "universal bound" for the Poincaré problem. This is precisely what happens in two classical counterexamples to be discussed in Section 6: the foliations of degree 1 on $\mathbb{P}_{\mathbb{C}}^{2}$ given by $\omega=\mathrm{d}\left(x^{p} z^{q-p} / y^{q}\right)$, with $p<q$, and the pencil of Lins Neto [20], a family of foliations of degree 4 admitting rational first integrals with unbounded degrees. In the first case, the typical fiber has a local branch at a dicritical singularity crossing a dicritical component of valence two. In the second family, the generic fiber repeatedly crosses radial singularities in a number of times which is unbounded within the family.

In Section 7 we study topologically bounded invariants of local foliations - those bounded by a function of the Milnor number. The central result of this section Theorem D - states that a local curve of separatrices that contains, besides the isolated separatrices, one separatrix attached to each dicritical component of valence one and a fixed number of separatrices attached to dicritical components of valence three or higher - separatrices crossing components of valence two are forbidden - has a reduction process whose length is topologically bounded, the same being true for the algebraic 
multiplicity. This result is sharp, as shown by the example $p x \mathrm{~d} y-q y \mathrm{~d} x=0$ with $p, q \in \mathbb{Z}^{+}$coprime. Here, the Milnor number is one and curves of separatrices - with a single branch passing by a component of valence two, when $q>p>1$, or with two branches passing through a dicritical component of valence one, when $q>p=1$ - may be obtained with reduction trees of arbitrarily large length. Returning to the Poincaré problem, in Theorem $\mathrm{E}$ we use the inequality of Theorem $\mathrm{C}$ in order to prove the existence of a bound for the Poincaré problem whenever the local branches of the algebraic curve $S$ are subject to the conditions of topological boundedness of Theorem D. This result especially indicates that the classical counterexamples for the Poincaré problem just mentioned offer essentially the two ways to violate the existence of a bound: either by means of highly degenerated separatrices crossing dicritical components of valences one or two, or through a multiple branched curve of separatrices attached to dicritical components of other valences.

We close this article with Section 8, where we apply local polar invariants in a result on the topological invariance of the algebraic multiplicity of a foliation. In Theorem $\mathrm{F}$ we prove that, for local foliations at $\left(\mathbb{C}^{2}, 0\right)$ having only convergent separatrices, the property of being second class and the algebraic multiplicity are topological invariants. This extends similar results in [5], for generalized curve foliations, and in [22], for nondicritical second class foliations.

\section{Basic notions and notation.}

A germ of formal foliation $\widehat{\mathcal{F}}$ in $\mathbb{C}^{2}$ is the object defined by a germ of formal 1 -form at $0 \in \mathbb{C}^{2}$

$$
\hat{\omega}=\hat{a}(x, y) \mathrm{d} x+\hat{b}(x, y) \mathrm{d} y,
$$

where $\hat{a}, \hat{b} \in \mathbb{C}[[x, y]]$. A separatrix for $\widehat{\mathcal{F}}$ is a germ of formal irreducible invariant curve. If $S$ is defined by a formal equation $\hat{f}$, then the invariance condition is expressed algebraically as

$$
\hat{f} \text { divides } \hat{\omega} \wedge \mathrm{d} \hat{f} \text { in } \mathbb{C}[[x, y]] \text {. }
$$

A formal foliation is said to be non-dicritical when it has finitely many separatrices. From now on, $E:(\mathcal{M}, \mathcal{D}) \rightarrow\left(\mathbb{C}^{2}, 0\right)$ stands for the process of reduction of singularities or desingularization of $\widehat{\mathcal{F}}$ (see [29] and also [5]), obtained by a finite sequence of punctual blowing-ups having $\mathcal{D}=E^{-1}(0)$ as the exceptional divisor, formed by a finite union of projective lines with normal crossings. In this process, all separatrices of $\widehat{\mathcal{F}}$ become smooth, disjoint and transverse to $\mathcal{D}$, none of them passing though a corner. Besides, the singularities along $\mathcal{D}$ of the pull-back foliation $E^{*} \widehat{\mathcal{F}}$ become reduced or simple, which means that, under some local formal change of coordinates, their linear parts belong to the following list:

(i) $y \mathrm{~d} x-\lambda x \mathrm{~d} y, \quad \lambda \notin \mathbb{Q}^{+}$;

(ii) $x \mathrm{~d} y$. 
In the first case - which is referred to as non-degenerate-, there are two formal separatrices tangent to the axes $\{x=0\}$ and $\{y=0\}$. In the convergent category, both separatrices converge. In the second case, the singularity is said to be a saddle-node. The formal normal form for such a singularity is given in [22]: up to a formal change of coordinates, the singularity is given by a 1 -form of the type

$$
\left(\zeta x^{k}-k\right) y \mathrm{~d} x+x^{k+1} \mathrm{~d} y, \quad k \in \mathbb{N}^{*}, \zeta \in \mathbb{C} .
$$

The invariant curve $\{x=0\}$ is called strong invariant curve while $\{y=0\}$ is the weak one. In the convergent category, the strong separatrix always converges whereas the weak separatrix may be purely formal, as in the famous example of Euler

$$
(x-y) \mathrm{d} x+x^{2} \mathrm{~d} y,
$$

where $y(x)=\sum_{n \geq 1}(n-1) ! x^{n}$ is the Taylor expansion of the weak invariant curve. The integer $k+1>1$ will be called weak index of the saddle-node.

If, in the reduction process, the germ of the divisor $\mathcal{D}$ happens to contain the weak invariant curve of some saddle-node, then the singularity is said to be a tangent saddlenode. A non-tangent saddle-node is also said to be well-oriented with respect to $\mathcal{D}$. We will denote by $\mathrm{T}(\widehat{\mathcal{F}})$ the set of all tangent saddle-nodes of $\widehat{\mathcal{F}}$. Following [22], we say that the foliation $\widehat{\mathcal{F}}$ is in the second class or is of second type when none of the singularities of $E^{*} \widehat{\mathcal{F}}$ over $\mathcal{D}$ are tangent saddle-nodes.

Let $\widehat{\mathcal{F}}$ be a germ of foliation having $(S, p)$ as a germ of formal smooth invariant curve. Take local coordinates $(x, y)$ in which $S$ is the curve $\{y=0\}$ and $p$ the point $(0,0)$. Let $\omega=\hat{a}(x, y) \mathrm{d} x+\hat{b}(x, y) \mathrm{d} y$ be a defining 1 -form for $\hat{\mathcal{F}}$. The integer $\operatorname{ord}_{0} \hat{b}(x, 0)$ is called the tangency index of $\widehat{\mathcal{F}}$ at $p$ with respect to $S$ and is $\operatorname{denoted}$ by $\operatorname{Ind}(\widehat{\mathcal{F}}, S, p)$. This is an invariant associated to $\widehat{\mathcal{F}}$ and $S$, independent of the choices made. If $S$ is the weak separatrix of a saddle-node, then $\operatorname{Ind}(\widehat{\mathcal{F}}, S, p)>1$ is precisely the weak index. On the other hand, if $S$ is either the strong separatrix of a saddle-node or a separatrix of a non-degenerate reduced singularity, then $\operatorname{Ind}(\widehat{\mathcal{F}}, S, p)=1$.

In the exceptional divisor $\mathcal{D}$, we denote by $\operatorname{Dic}(\widehat{\mathcal{F}})$ the set of dicritical components, comprising all projective lines generically transverse to $E^{*} \widehat{\mathcal{F}}$. A separatrix $S$ of $\widehat{\mathcal{F}}$ is said to be isolated if its strict transform $E^{*} S$ does not meet a dicritical component. This concept is well defined as long as we fix a minimal reduction of singularities for $\widehat{\mathcal{F}}$ see that, in the definition of reduction of singularities, there are also conditions on the desingularization of the separatrices. We denote by $\mathcal{I}(\widehat{\mathcal{F}})$ the set of isolated separatrices. On the other hand, a separatrix whose strict transform crosses a dicritical component is called a curvet. The set of all curvets associated to $D \in \operatorname{Dic}(\widehat{\mathcal{F}})$ is denoted by $\operatorname{Curv}(D)$. Finally, $\mathrm{v}(D)$ stands for the valence of $D \in \operatorname{Dic}(\widehat{\mathcal{F}})$, defined as the number of components of $\mathcal{D}$ intersecting $D$, other from $D$ itself.

REMARK 2.1. The above definitions can be formulated in the convergent category and it may seem somewhat strange to introduce them in the formal category. Indeed, for convergent foliations, the natural and geometric notion of leaves does exist whereas only the notion of separatrix makes sense in the formal setting. However, the interest and the need to work with formal objects will be evident as soon as the concept of balanced 
equation is defined.

\section{Balanced equation of separatrices.}

With a slight change in the definition, we follow [18] in the concept below.

DEFINITION 3.1. A A balanced equation of separatrices for a germ of formal singular foliation in $\left(\mathbb{C}^{2}, 0\right)$ is a formal meromorphic function $\hat{F}$ whose divisor has the form

$$
(\hat{F})_{0}-(\hat{F})_{\infty}=\sum_{C \in \mathcal{I}(\widehat{\mathcal{F}})}(C)+\sum_{D \in \operatorname{Dic}(\widehat{\mathcal{F}})} \sum_{C \in \operatorname{Curv}(D)} a_{D, C}(C),
$$

where, for every dicritical component $D \subset \mathcal{D}$, the coefficients $a_{D, C} \in\{-1,0,1\}$ are zero except for finitely many $C \in \operatorname{Curv}(D)$ and satisfy the following equality:

$$
\sum_{C \in \operatorname{Curv}(D)} a_{D, C}=2-\mathrm{v}(D)
$$

The balanced equation of separatrices $\hat{F}$ is said to be adapted to a curve of separatrices $C$ if $C \subset(\hat{F})_{0}$.

Since $a_{D, C}$ belongs to $\{-1,0,1\}$, the function $\hat{F}$ has reduced zeros and poles without multiplicities. For instance, the radial foliation given by $x \mathrm{~d} y-y \mathrm{~d} x$ has only one dicritical component whose valence is 0 . Thus $F=x y$ is a balanced equation of separatrices. However, $F=x y(x-y) /(x+y)$ is also a balanced equation, adapted to the curve $C=\{x-y=0\}$. If $\widehat{\mathcal{F}}$ is non-dicritical, then a balanced equation is nothing but any equation of the finite set of separatrices.

We recall some basic facts about balanced equations of separatrices established in [18]. First a definition:

Definition 3.2. Let $\widehat{\mathcal{F}}$ be a formal foliation at $\left(\mathbb{C}^{2}, 0\right)$ and $E:(\mathcal{M}, \mathcal{D}) \rightarrow\left(\mathbb{C}^{2}, 0\right)$ be a minimal process of reduction of singularities. The tangency excess of $\widehat{\mathcal{F}}$ along $\mathcal{D}$ is the number

$$
\tau(\widehat{\mathcal{F}})=\sum_{q \in \mathrm{T}\left(E^{*} \widehat{\mathcal{F}}\right)} \sum_{D \in V(q)} \rho(D)\left(\operatorname{Ind}\left(E^{*} \widehat{\mathcal{F}}, D, q\right)-1\right),
$$

where $q$ runs over all the tangent saddle-nodes of $E^{*} \widehat{\mathcal{F}}$ and $V(q)$ stands for the set of irreducible components of $\mathcal{D}$ containing the point $q$. The number $\rho(D)$ stands for the multiplicity of $D$, which coincides with the algebraic multiplicity of a curve $\gamma$ at $\left(\mathbb{C}^{2}, 0\right)$ such that $E^{*} \gamma$ is transversal to $D$ outside a corner of $\mathcal{D}$.

It is clear that $\tau(\widehat{\mathcal{F}}) \geq 0$. Besides, $\tau(\widehat{\mathcal{F}})=0$ if and only if there are no tangent saddle-nodes in the reduction of singularities of $\widehat{\mathcal{F}}$, that is, if and only if $\widehat{\mathcal{F}}$ is a foliation of second type. Having the definition in mind, the following fact is proved in $[\mathbf{1 8}]$ :

Proposition 3.3. Let $\hat{F}$ be a balanced equation of separatrices for the formal 
foliation $\widehat{\mathcal{F}}$. Denote by $\nu_{0}(\hat{F})$ and $\nu_{0}(\widehat{\mathcal{F}})$ their algebraic multiplicities. Then

$$
\nu_{0}(\widehat{\mathcal{F}})=\nu_{0}(\hat{F})-1+\tau(\widehat{\mathcal{F}}) .
$$

Inspired in this result, if $\hat{F}$ is a balanced equation of separatrices for the foliation $\widehat{\mathcal{F}}$, we define the number

$$
\nu_{0}^{*}(\widehat{\mathcal{F}})=\nu_{0}(\hat{F})-1
$$

and call it the pure algebraic multiplicity of $\widehat{\mathcal{F}}$ at $0 \in \mathbb{C}^{2}$. This invariant assembles the contribution to the algebraic multiplicity given by the separatrices and the reduction structure - by which we mean the combinatory of the desingularization tree along with the position of the dicritical components - , but discarding the contribution given by the tangent saddles-nodes. Evidently, $\nu_{0}^{*}(\widehat{\mathcal{F}}) \leq \nu_{0}(\widehat{\mathcal{F}})$, equality holding if and only if $\widehat{\mathcal{F}}$ is of second type. The number $\nu_{0}^{*}(\widehat{\mathcal{F}})$ would turn out to be the algebraic multiplicity of some second type foliation sharing with $\widehat{\mathcal{F}}$ the same reduction structure and the same balanced equation. However, we cannot assure the existence of such a foliation and we do not know if $\nu_{0}^{*}(\widehat{\mathcal{F}})$ is realizable as an algebraic multiplicity in this way. Nevertheless, we can prove the following:

Lemma 3.4. We have $\nu_{0}^{*}(\widehat{\mathcal{F}}) \geq 0$. The inequality is strict when $\widehat{\mathcal{F}}$ is dicritical.

Proof. We have to proof that, if $\hat{F}$ is a balanced equation of separatrices for $\widehat{\mathcal{F}}$, then $\nu_{0}(\hat{F}) \geq 1$, the equality being strict in the dicritical case. The result is obvious if $\widehat{\mathcal{F}}$ is non-dicritical, since the balanced equation has no poles and a separatrix always exits by the Separatrix Theorem $[6]$. For the dicritical case, let $E:(\mathcal{M}, \mathcal{D}) \rightarrow\left(\mathbb{C}^{2}, 0\right)$ be a minimal reduction process for $\widehat{\mathcal{F}}$. The proof relies on the fact that through each connected component of the invariant part of the divisor $\mathcal{D}$ passes at least one separatrix of $\widehat{\mathcal{F}}$ (see [26]). Let us start by supposing that $\mathcal{D}$ contains only dicritical components with valence up to two. Then $\hat{F}$ is devoid of poles and $\nu_{0}(\hat{F}) \geq 0$. Aside from the trivial case $\mathcal{D}=D$ with $\mathrm{v}(D)=0$, for which $\nu_{0}(\hat{F})=2$, we get $\nu_{0}(\hat{F})>1$ in the two following cases:

(i) There exists a dicritical component $D \in \mathcal{D}$ with $\mathrm{v}(D)=1$; here, $\hat{F}$ contains a separatrix crossing $D$ and there exists at least one isolated separatrix crossing the invariant part of $\mathcal{D}$.

(ii) There exists a dicritical component $D \in \mathcal{D}$ with $\mathrm{v}(D)=2$; then $D$ disconnects the invariant part of $\mathcal{D}$ giving rise to two connected components, where we find at least two isolated separatrices.

Now, we admit the existence of a dicritical component $D \subset \mathcal{D}$ of valence $\mathrm{v}(D) \geq 3$. In the course of the reduction process, $D$ appears as the result of a blowing-up at $0 \in \mathbb{C}^{2}$, at non-corner point or at a corner point. Corresponding to these three cases, new sequences of blowing-ups will be needed starting at, respectively, $\mathrm{v}(D), \mathrm{v}(D)-1$ or $\mathrm{v}(D)-2$ points of $D$. Each of these points gives rise to at least to one isolated separatrix with algebraic multiplicity no less than that of any dicritical separatrix crossing $D$. Therefore, the 
negative contribution to $\nu_{0}(\hat{F})$ given by the $\mathrm{v}(D)-2$ dicritical separatrices attached to $D$ will be neutralized by the contribution of these isolated separatrices. Now, in order to see that $\nu_{0}(\hat{F})>1$, it suffices to take $D$ as the first dicritical component with $\mathrm{v}(D) \geq 3$ appearing in the reduction process. The extra separatrices we need are found using the arguments of cases (i) and (ii) above.

REMARK 3.5. In the non-dicritical case, we may have $\nu_{0}^{*}(\hat{F})=0$, even if $0 \in \mathbb{C}^{2}$ is a singularity of $\widehat{\mathcal{F}}$. For instance, the local foliation $\widehat{\mathcal{F}}$ given by the non-linearizable Poincaré-Dulac normal form $\omega=\left(n x+\zeta y^{n}\right) d y-y d x$, where $\zeta \neq 0$ and $n \in \mathbb{N}$ with $n \geq 2$, has $y=0$ as the unique separatrix, giving $\nu_{0}(\hat{F})=1$. Another example is given by the nilpotent singularity by $\eta=d\left(y^{2}+x^{4}\right)+4 x^{2} d y$, whose unique separatrix is $y=-x^{2}$.

Definition 3.6. Let $\widehat{\mathcal{F}}$ be a local foliation at $\left(\mathbb{C}^{2}, 0\right)$ and $E:(\mathcal{M}, \mathcal{D}) \rightarrow\left(\mathbb{C}^{2}, 0\right)$ be a minimal process of reduction of singularities. If $D \subset \mathcal{D}$ is an irreducible component, the valuation of $\widehat{\mathcal{F}}$ along $D$, denoted by $\nu_{D}(\widehat{\mathcal{F}})$, is the order of vanishing of $E^{*} \omega$ along $D$, where $\omega$ is any 1 -form inducing $\widehat{\mathcal{F}}$.

For instance, if $D$ arises from the first blowing-up at $0 \in \mathbb{C}^{2}$, then

$$
\nu_{D}(\widehat{\mathcal{F}})=\left\{\begin{array}{l}
\nu_{0}(\widehat{\mathcal{F}}) \text { if } D \text { is non-dicritical } \\
\nu_{0}(\widehat{\mathcal{F}})+1 \text { if } D \text { is dicritical. }
\end{array}\right.
$$

In much the same way, if $\hat{F}$ is a formal meromorphic function at $\left(\mathbb{C}^{2}, 0\right)$, then, given some process of reduction of singularities $E:(\mathcal{M}, \mathcal{D}) \rightarrow\left(\mathbb{C}^{2}, 0\right)$, we can define the valuation of $\hat{F}$ along $D$, denoted by $\nu_{D}(\hat{F})$, as the order of vanishing of $E^{*} F=F \circ E$ along $D$. The following fact also appears in $[\mathbf{1 8}]$ :

Proposition 3.7. Let $\widehat{\mathcal{F}}$ be a foliation of second type having $\hat{F}$ as a balanced equation of separatrices. Let $E:(\mathcal{M}, \mathcal{D}) \rightarrow\left(\mathbb{C}^{2}, 0\right)$ be a minimal process of reduction of singularities for $\widehat{\mathcal{F}}$. Then, for every component $D \in \mathcal{D}$, it holds

$$
\nu_{D}(\hat{F})=\left\{\begin{array}{l}
\nu_{D}(\widehat{\mathcal{F}})+1 \quad \text { if } D \text { is non-dicritical } \\
\nu_{D}(\widehat{\mathcal{F}}) \text { if } D \text { is dicritical. }
\end{array}\right.
$$

In particular, $\nu_{D}(\hat{F})>0$ for all $D \in \mathcal{D}$.

Let $\widehat{\mathcal{F}}$ be a foliation, not necessarily of second type, with balanced equation of separatrices $\hat{F}$ and minimal reduction process $E:(\mathcal{M}, \mathcal{D}) \rightarrow\left(\mathbb{C}^{2}, 0\right)$. Following what was done for the algebraic multiplicity, we associate to $\widehat{\mathcal{F}}$ an invariant $\nu_{D}^{*}(\widehat{\mathcal{F}})$ called pure valuation along $D \in \mathcal{D}$. As before, this would be the valuation $\nu_{D}(\widehat{\mathcal{G}})$ for some foliation $\widehat{\mathcal{G}}$ of second type sharing with $\widehat{\mathcal{F}}$ the same reduction structure and the same balanced equation, if such a $\widehat{\mathcal{G}}$ existed. Since this is not guaranteed, we appeal to the following recursive definition: if $D$ arises from the first blowing-up at $0 \in \mathbb{C}^{2}$, then

$$
\nu_{D}^{*}(\widehat{\mathcal{F}})=\left\{\begin{array}{l}
\nu_{0}^{*}(\widehat{\mathcal{F}}) \text { if } D \text { is non-dicritical } \\
\nu_{0}^{*}(\widehat{\mathcal{F}})+1 \quad \text { if } D \text { is dicritical. }
\end{array}\right.
$$


In order to proceed to the inductive step, write $E=E_{k} \circ \cdots \circ E_{1}$ the divided blowingup, where $k \geq 2$, and, for $1 \leq j \leq k$, let $D_{j}$ be the divisor associated to $E_{j}$ and $\widehat{\mathcal{F}}_{j}=\left(E_{j} \circ \cdots \circ E_{1}\right)^{*} \widehat{\mathcal{F}}$ be the strict transform foliation. Let $1 \leq i<k$ and suppose that the pure valuation is defined for irreducible components appearing at the height $i$. Suppose that $E_{i+1}$ is a blowing-up at a point $p \in \mathcal{D}_{i}=\cup_{j=1}^{i} D_{j}$. We define:

$$
\nu_{D_{i+1}}^{*}(\widehat{\mathcal{F}})=\nu_{p}^{*}\left(\widehat{\mathcal{F}}_{i}\right)+\left(1-\epsilon\left(D_{i+1}\right)\right)+\sum_{D \in V(p)} \nu_{D}^{*}(\widehat{\mathcal{F}}),
$$

where $V(p)$ stands for the set of irreducible components of $\mathcal{D}_{i}$ containing $p$ and, for a component $D \subset \mathcal{D}$,

$$
\epsilon(D)= \begin{cases}1 & \text { if } D \text { is non-dicritical } \\ 0 & \text { if } D \text { is dicritical }\end{cases}
$$

Notice that, as a consequence of Lemma 3.4, except for the case of a non-dicritical foliation with a unique smooth separatrix, $\nu_{D}^{*}(\widehat{\mathcal{F}})>0$ for every $D \in \mathcal{D}$. Formula $(3.3)$ is satisfied by the usual valuation. This is the key for the inductive step in the proof of Proposition 3.7 in [18]. The same arguments therein give us a new version for this result:

Proposition 3.8. Let $\widehat{\mathcal{F}}$ be a foliation having $\hat{F}$ as a balanced equation of separatrices. Let $E:(\mathcal{M}, \mathcal{D}) \rightarrow\left(\mathbb{C}^{2}, 0\right)$ be a minimal process of reduction of singularities for $\widehat{\mathcal{F}}$. Then, for every component $D \in \mathcal{D}$, it holds

$$
\nu_{D}(\hat{F})=\left\{\begin{array}{l}
\nu_{D}^{*}(\widehat{\mathcal{F}})+1 \quad \text { if } D \text { is non-dicritical } \\
\nu_{D}^{*}(\widehat{\mathcal{F}}) \quad \text { if } D \text { is dicritical. }
\end{array}\right.
$$

In particular, $\nu_{D}(\hat{F})>0$ for all $D \in \mathcal{D}$.

When $\widehat{\mathcal{F}}$ is non-dicritical and of second type, it is well known (see [22], Corollary 3.1.10) that $\widehat{\mathcal{F}}$ and $\mathrm{d} \hat{F}$ share the same process of reduction of singularities. However, this fails to be true when $\widehat{\mathcal{F}}$ is dicritical. For instance, the quasi-radial foliation given by $p x \mathrm{~d} y-q y \mathrm{~d} x$, with $p, q \in \mathbb{N}^{*}$ relatively prime, admits a balanced equation whose differential is $\mathrm{d} \hat{F}=\mathrm{d}(x y)=x \mathrm{~d} y+y \mathrm{~d} x$. The latter is reduced whereas $p x \mathrm{~d} y-q y \mathrm{~d} x$ needs a reduction process attached to the Euclid's algorithm of the pair $(p, q)$. Nevertheless, we can establish the following link between the two reduction processes:

Proposition 3.9. Let $\widehat{\mathcal{F}}$ be a foliation having $\hat{F}$ as a balanced equation of separatrices. Let $E$ be the reduction process of $\widehat{\mathcal{F}}$. Then:

(a) Any component $D \subset E^{-1}(0)$ is invariant by $E^{*} \mathrm{~d} \hat{F}$.

(b) Any singularity of $E^{*} \mathrm{~d} \hat{F}$ is reduced except possibly along the dicritical components of $\widehat{\mathcal{F}}$, where $E^{*} \mathrm{~d} \hat{F}$ might have dicritical singularities.

Proof. The proof relies on Proposition 3.8. At a point $p \in D$ there are local coordinates $(x, y)$ such that the pull-back of $\hat{F}$ is written in the following way: 
(1) if $p$ is neither a zero nor a pole of the strict transform of $\hat{F}$, then $\left(E^{*} \hat{F}\right)_{p}=x^{\nu_{D}(\hat{F})}$, where $x$ is a local equation for $D$ near $p$.

(2) if $p$ is a corner, say $p=D_{1} \cap D_{2}$, then $\left(E^{*} \hat{F}\right)_{p}=x^{\nu_{D_{1}}(\hat{F})} y^{\nu_{D_{2}}(\hat{F})}$, where $x$ and $y$ are local equations for $D_{1}$ and $D_{2}$, respectively.

(3) if $p$ is either a zero or a pole of the strict transform of $\hat{F}$, then $\left(E^{*} \hat{F}\right)_{p}$ is either $x^{\nu_{D}(\hat{F})} y$ or $x^{\nu_{D}(\hat{F})} / y$, where $x$ is a local equation for $D$ and $y$ a local equation for the zero or the pole.

The combination of the above remarks with the upcoming lemma yields the proposition.

LEMMA 3.10. Let $\hat{H}$ be any meromorphic function, $E$ be any blowing-up process and $D$ be any component of the exceptional divisor. Then $D$ is dicritical for $E^{*} \mathrm{~d} \hat{H}$ if and only if $\nu_{D}(\hat{H})=0$.

Indeed, according to Proposition 3.8, if $D \subset E^{-1}(0)$ then $\nu_{D}(\hat{F})$ is strictly positive. Thus, any component of the exceptional divisor of $E$ is invariant. Moreover, around the points of intersection of the poles of $\hat{F}$ with the divisor, there are local coordinates $(x, y)$ such that $\left(E^{*} \hat{F}\right)=x^{N} / y$, which is a dicritical singularity for $E^{*} \mathrm{~d} \hat{F}$, reduced after $N$ blowing-ups.

Even when $\widehat{\mathcal{F}}$ is convergent, it is not enough to consider a balanced equation formed only by convergent invariant curves. Of course, formal separatrices may appear as weak invariant curves of saddle-nodes. For instance, a balanced equation for the Euler singularity is given by the formal equation

$$
\hat{F}=x\left(y-\sum_{n \geq 1}(n-1) ! x^{n}\right) .
$$

Evidently, for a convergent foliation $\widehat{\mathcal{F}}$, all possible formal separatrices are isolated, the ones in $\operatorname{Dic}(\widehat{\mathcal{F}})$ being all convergent.

One of the main features of balanced equations is their good behavior under blowingups:

Lemma 3.11. Let $\hat{F}$ be a balanced equation of separatrices for $\widehat{\mathcal{F}}$. Let $\pi:\left(\widetilde{\mathbb{C}^{2}}, D\right) \rightarrow$ $\left(\mathbb{C}^{2}, 0\right)$ be the standard blowing-up at the origin. Then, for any point $p \in D$, singular for $\pi^{*} \widehat{\mathcal{F}}$, the germ at $p$ of the meromorphic function

$$
\frac{\hat{F} \circ \pi}{h_{p}^{\nu_{0}(\hat{F})-\epsilon(\widehat{\mathcal{F}})},}
$$

where $h_{p}$ is a local equation of $D$ and $\epsilon(\widehat{\mathcal{F}})=\epsilon(D)$ is defined in (3.4), is a balanced equation for the germ of $\pi^{*} \widehat{\mathcal{F}}$ at $p$.

Proof. We examine separately the non-dicritical and the dicritical cases. 
First case: $D$ is non-dicritical. We consider the reduction of singularities of $\pi^{*} \widehat{\mathcal{F}}$ at $p$ as part of that of $\widehat{\mathcal{F}}$. From the point of view of $\pi^{*} \widehat{\mathcal{F}}$, the germ of $D$ at $p$ is no longer part of the divisor, turning into a separatrix. The matter is to decide whether it is an isolated or a dicritical separatrix. Let $D^{\prime}$ be the component of the desingularization divisor of $\pi^{*} \widehat{\mathcal{F}}$ intersecting $D$. If $D^{\prime}$ is non-dicritical, then the germ of $D$ is an isolated separatrix for $\pi^{*} \widehat{\mathcal{F}}$. Thus,

$$
\frac{\hat{F} \circ \pi}{h_{p}^{\nu_{0}(\hat{F})}} h_{p}
$$

is a balanced equation for $\pi^{*} \widehat{\mathcal{F}}$ at $p$. On the other hand, if $D^{\prime}$ is dicritical, then its valence as a dicritical component of $\pi^{*} \widehat{\mathcal{F}}$ at $p$ is one unit less its valence as a dicritical component of $\widehat{\mathcal{F}}$. Thus, in view of (3.1), equation (3.5) is again a balanced equation for $\pi^{*} \widehat{\mathcal{F}}$ at $p$.

Second case: $D$ is dicritical. Then, by the definition of reduction of singularities, the component $D^{\prime}$ touching $D$ in the reduction of singularities of $\widehat{\mathcal{F}}$ cannot be dicritical. Since $D$ is not $\pi^{*} \widehat{\mathcal{F}}$-invariant, $\hat{F} \circ \pi / h_{p}^{\nu_{0}(F)}$ is a balanced equation of $\pi^{*} \widehat{\mathcal{F}}$ at $p$.

This lemma is the key ingredient for most of the properties to be proved in this article, allowing us to reason inductively on the length of the reduction process.

\section{Polar intersection and polar excess.}

Let $\hat{\eta}$ be a formal meromorphic 1-form defined near a point $p$ of a complex surface. It can be regarded as a 1 -form at $\left(\mathbb{C}^{2}, 0\right)$ by taking analytic coordinates $(x, y)$ for which $p=(0,0)$, so that

$$
\hat{\eta}=\frac{\hat{\omega}}{H}=\frac{P \mathrm{~d} x+Q \mathrm{~d} y}{H},
$$

where $P, Q$ and $H$ are formal functions in $\mathbb{C}[[x, y]]$. For $(a: b) \in \mathbb{P}_{\mathbb{C}}^{1}$, the polar curve of $\hat{\eta}$ with respect to $(a: b)$ is the curve $\mathcal{P}_{(a: b)}^{\hat{\eta}}$ with formal meromorphic equation $(a P+b Q) / H$. In the convergent case, when $a \neq 0$, the points of $\mathcal{P}_{(a: b)}^{\hat{\eta}}$ outside $(H)_{0}$ are those where $\hat{\eta}$ defines a tangent line with inclination $b / a$. Now, suppose that $\hat{B}$ is an irreducible curve invariant by $\hat{\eta}$ such that $\hat{B} \nsubseteq(\hat{\eta})_{\infty}=(H)_{0}$, having $\gamma(t)$ as a formal Puiseux parametrization. We calculate the intersection number

$$
\left(\mathcal{P}_{(a: b)}^{\hat{\eta}}, \hat{B}\right)_{p}=\operatorname{ord}_{t=0}\left(\frac{a P+b Q}{H} \circ \gamma\right),
$$

which does not depend on the choice of coordinates $(x, y)$. This enables us to set the following definition:

DEFinition 4.1. The polar intersection number of $\hat{\eta}$ and $\hat{B}$ at $p$ is the integer

$$
\left(\mathcal{P}^{\hat{\eta}}, \hat{B}\right)_{p}=\left(\mathcal{P}_{(a: b)}^{\hat{\eta}}, \hat{B}\right)_{p}
$$


obtained for a generic point $(a: b) \in \mathbb{P}_{\mathbb{C}}^{1}$.

This is an adaptation, for formal meromorphic forms, of the definition in [9]. Clearly, polar intersection numbers are well defined, independent both of the coordinates and of the choice of the Puiseux parametrization of $B$.

Polar intersection numbers have a nice behavior under blowing-ups:

LEMma 4.2. Let $\hat{\eta}$ be a formal meromorphic 1 -form at $p \in \mathbb{C}^{2}$ and $\hat{B}$ be an irreducible invariant curve as in the previous definition. Let $\pi:\left(\widetilde{\mathbb{C}^{2}}, D\right) \rightarrow\left(\mathbb{C}^{2}, p\right)$ be the standard blowing-up at $p$. Denote by $\tilde{B}$ the strict transform of $\hat{B}$ and $q=\tilde{B} \cap D$. Then

$$
\left(\mathcal{P}^{\pi^{*} \hat{\eta}}, \tilde{B}\right)_{q}=\left(\mathcal{P}^{\hat{\eta}}, \hat{B}\right)_{p}+\nu_{q}(\tilde{B})
$$

Proof. Let us fix coordinates $(x, y)$ and a Puiseux parametrization $\gamma(t)=$ $(x(t), y(t))$ of $\hat{B}$ with $\nu_{p}(\hat{B})=\operatorname{ord}_{t=0} x(t)$. Let us write $\pi(x, u)=(x, u x)$. In these coordinates, $\tilde{B}$ is parametrized by $\tilde{\gamma}(t)=(x(t), u(t)=y(t) / x(t))$. We have

$$
\pi^{*} \hat{\eta}=\frac{1}{H(x, u x)}((P(x, u x)+u Q(x, u x)) \mathrm{d} x+x Q(x, u x) \mathrm{d} u) .
$$

For $(a: b) \in \mathbb{P}_{\mathbb{C}}^{1}$, we make the following computation:

$$
\begin{aligned}
\left(\mathcal{P}_{(a: b)}^{\pi^{*} \hat{n}}, \tilde{B}\right)_{q}= & \operatorname{ord}_{t=0}\left(\frac{a(P(x, u x)+u Q(x, u x))+b x Q(x, u x)}{H(x, u x)} \circ \tilde{\gamma}\right) \\
= & \operatorname{ord}_{t=0}\left(\frac{a(P \circ \gamma(t)+u(t) Q \circ \gamma(t))+b x(t) Q \circ \gamma(t)}{H \circ \gamma(t)}\right) \\
= & \min \left\{\operatorname{ord}_{t=0}(P \circ \gamma(t)+u(t) Q \circ \gamma(t)), \operatorname{ord}_{t=0}(x(t) Q \circ \gamma(t))\right\} \\
& -\operatorname{ord}_{t=0} H \circ \gamma(t) .
\end{aligned}
$$

Now, since $\hat{B}$ is invariant, we have $P \circ \gamma(t) x^{\prime}(t)+Q \circ \gamma(t) y^{\prime}(t)=0$. Noting that $\operatorname{ord}_{t=0} x(t) \leq \operatorname{ord}_{t=0} y(t)$, this gives in particular that $\operatorname{ord}_{t=0} Q \circ \gamma(t) \leq \operatorname{ord}_{t=0} P \circ \gamma(t)$ and therefore $\left(\mathcal{P}_{(a: b)}^{\hat{\eta}}, \hat{B}\right)_{p}=\operatorname{ord}_{t=0} Q \circ \gamma(t)-\operatorname{ord}_{t=0} H \circ \gamma(t)$. It is straightforward that

$$
\begin{aligned}
\operatorname{ord}_{t=0}(P \circ \gamma(t)+u(t) Q \circ \gamma(t)) & =\operatorname{ord}_{t=0}\left(\frac{-u^{\prime}(t) x(t)}{x^{\prime}(t)} Q \circ \gamma(t)\right) \\
& =\operatorname{ord}_{t=0} u(t)+\operatorname{ord}_{t=0} Q \circ \gamma(t) .
\end{aligned}
$$

We finally find

$$
\begin{aligned}
\left(\mathcal{P}_{(a: b)}^{\pi^{*} \hat{n}}, \tilde{B}\right)_{q}= & \min \left\{\operatorname{ord}_{t=0} u(t)+\operatorname{ord}_{t=0} Q \circ \gamma(t), \operatorname{ord}_{t=0} x(t)+\operatorname{ord}_{t=0} Q \circ \gamma(t)\right\} \\
& -\operatorname{ord}_{t=0} H \circ \gamma(t) \\
= & \min \left\{\operatorname{ord}_{t=0} u(t), \operatorname{ord}_{t=0} x(t)\right\}+\operatorname{ord}_{t=0} Q \circ \gamma(t)-\operatorname{ord}_{t=0} H \circ \gamma(t) \\
= & \nu_{q}(\tilde{B})+\left(\mathcal{P}_{(a: b)}^{\hat{\eta}}, \hat{B}\right)_{p} .
\end{aligned}
$$

The proof is finished by taking generic $(a: b) \in \mathbb{P}_{\mathbb{C}}^{1}$. 
Our interest does not lie in the absolute values of polar intersection numbers. The idea is to compare polar intersection numbers of a foliation and a "reference foliation" having the balanced equation as a first integral. This is the same principle developed in $[\mathbf{9}]$ for the non-dicritical case. There, however, the finiteness of the set of separatrices gives a straight choice for this reference foliation. More specifically, we define the following invariant:

Definition 4.3. Let $\widehat{\mathcal{F}}$ be a germ of singular foliation at a point $p \in \mathbb{C}^{2}$ having $\hat{F}$ as a balanced equation of separatrices. Let $C \subset(\hat{F})_{0}$ be a union of zeros of $\hat{F}$ and consider the decomposition in irreducible components $C=\cup_{i=1}^{n} C_{i}$. We define the polar excess index of $\widehat{\mathcal{F}}$ with respect to the irreducible component $C_{i}$ as

$$
\Delta_{p}\left(\widehat{\mathcal{F}}, C_{i}\right)=\left(\mathcal{P}^{\widehat{\mathcal{F}}}, C_{i}\right)_{p}-\left(\mathcal{P}^{\mathrm{d} \hat{F}}, C_{i}\right)_{p}
$$

and with respect to the whole curve $C$ as

$$
\Delta_{p}(\widehat{\mathcal{F}}, C)=\sum_{i=1}^{n} \Delta_{p}\left(\widehat{\mathcal{F}}, C_{i}\right)
$$

where $\left(\mathcal{P}^{\mathrm{d} \hat{F}}, C_{i}\right)_{p}$ refer to the polar intersection numbers of the formal foliation defined by $\mathrm{d} \hat{F}$. We also introduce a relative version of the polar excess index: if $f$ is the formal equation of the curve of separatrices $C \subset(\hat{F})_{0}$ above, instead of using the whole balanced equation $\hat{F}=G / H$ as done in the calculation of the polar excess, we take $f / H$. More precisely:

$$
\Delta_{p}^{\mathrm{rel}}(\widehat{\mathcal{F}}, C)=\sum_{i=1}^{n}\left(\left(\mathcal{P}^{\widehat{\mathcal{F}}}, C_{i}\right)_{p}-\left(\mathcal{P}^{\mathrm{d}(f / H)}, C_{i}\right)_{p}\right)
$$

EXAMPLE 4.4. Let us see what is $\Delta_{p}(\widehat{\mathcal{F}}, B)$ for a branch of separatrix $B$ in the reduced case.

(1) $\widehat{\mathcal{F}}$ is non singular at $p$. In this case, $B$ is the local leaf at $p$ and it is easy to see that $\Delta_{p}(\widehat{\mathcal{F}}, B)=0$.

(2) $\widehat{\mathcal{F}}$ has a reduced non-degenerate singularity at $p$. Now we can take local coordinates such that $p=(0,0)$ and $\widehat{\mathcal{F}}$ is given by

$$
x(1+u(x, y)) \mathrm{d} y+y(\lambda+v(x, y)) \mathrm{d} y .
$$

The function $\hat{F}=x y$ is a balanced equation. If $B$ is either $x=0$ or $y=0$, it is straight that $\left(\mathcal{P}^{\widehat{\mathcal{F}}}, B\right)_{p}=1$ and also $\left(\mathcal{P}^{\mathrm{d} \hat{F}}, B\right)_{p}=1$. Therefore $\Delta_{p}(\widehat{\mathcal{F}}, B)=0$.

(3) $\widehat{\mathcal{F}}$ has a saddle-node at $p$. We take the formal coordinates $(x, y)$ inducing the normal form of equation (2.1):

$$
\left(\zeta x^{k}-k\right) y \mathrm{~d} x+x^{k+1} \mathrm{~d} y, \quad k \in \mathbb{N}^{*}, \zeta \in \mathbb{C} .
$$


Again, $\hat{F}=x y$ is a balanced equation. If $B$ is the strong separatrix, $x=0$, we find $\left(\mathcal{P}^{\widehat{\mathcal{F}}}, B\right)_{p}=1$ and, thus, $\Delta_{p}(\widehat{\mathcal{F}}, B)=0$. On the other hand, if $B$ is the weak separatrix, $y=0$, we have $\left(\mathcal{P}^{\widehat{\mathcal{F}}}, B\right)_{p}=k+1$, which results in $\Delta_{p}(\widehat{\mathcal{F}}, B)=k>0$.

Keeping the notation of Lemma 4.2, we have:

Proposition 4.5. Let $B$ be an irreducible component of $(\hat{F})_{0}$. Then

$$
\Delta_{p}(\widehat{\mathcal{F}}, B)=\Delta_{q}\left(\pi^{*} \widehat{\mathcal{F}}, \tilde{B}\right)+\tau(\widehat{\mathcal{F}}) \nu_{p}(B),
$$

where $\tau(\widehat{\mathcal{F}})$ is tangency excess of $\widehat{\mathcal{F}}$ defined in equation (3.2). In particular,

$$
\Delta_{p}(\widehat{\mathcal{F}}, B) \geq \Delta_{q}\left(\pi^{*} \widehat{\mathcal{F}}, \tilde{B}\right),
$$

equality holding if and only if $\widehat{\mathcal{F}}$ is of second type.

Proof. Consider adapted coordinates $(x, u)$ for which $\pi(x, u)=(x, u x)$ and $B$ is given by $\gamma(t)=(x(t), y(t))$, where $\nu_{p}(B)=\operatorname{ord}_{t=0} x(t)$. In order to avoid any confusion, let us denote by $\hat{F}_{p}$ the balanced equation for $\widehat{\mathcal{F}}$ at $p$ and $\hat{F}_{q}$ the balanced equation for $\pi^{*} \widehat{\mathcal{F}}$ at $q$. Following Lemma 3.11, the relation between the two balanced equations is

$$
\pi^{*} \hat{F}_{p}=x^{\nu_{p}\left(\hat{F}_{p}\right)-\epsilon(\widehat{\mathcal{F}})} \hat{F}_{q}
$$

Taking derivatives in the above relation, we get

$$
\pi^{*} \mathrm{~d} \hat{F}_{p}=x^{\nu_{p}\left(\hat{F}_{p}\right)-\epsilon(\widehat{\mathcal{F}})} \mathrm{d} \hat{F}_{q}+\left(\nu_{p}\left(\hat{F}_{p}\right)-\epsilon(\widehat{\mathcal{F}})\right) x^{\nu_{p}\left(\hat{F}_{p}\right)-\epsilon(\widehat{\mathcal{F}})-1} \hat{F}_{q} \mathrm{~d} x
$$

Since $\hat{F}_{q}$ vanishes along $\tilde{B}$, for a fixed $(a: b) \in \mathbb{P}_{\mathbb{C}}^{1}$ one has

$$
\left(\mathcal{P}_{(a: b)}^{\pi^{*} \mathrm{~d} \hat{F}_{p}}, \tilde{B}\right)_{q}=\operatorname{ord}_{t=0} x(t)^{\nu_{p}\left(\hat{F}_{p}\right)-\epsilon(\widehat{\mathcal{F}})}+\left(\mathcal{P}_{(a: b)}^{\mathrm{d} \hat{F}_{q}}, \tilde{B}\right)_{q} .
$$

Now, taking $(a: b) \in \mathbb{P}_{\mathbb{C}}^{1}$ generic and using Lemma 4.2, we obtain

$$
\left(\mathcal{P}^{\mathrm{d} \hat{F}_{q}}, \tilde{B}\right)_{q}=\left(\mathcal{P}^{\mathrm{d} \hat{F}_{p}}, B\right)_{p}-\left(\nu_{p}\left(\hat{F}_{p}\right)-\epsilon(\widehat{\mathcal{F}})\right) \nu_{p}(B)+\nu_{q}(\tilde{B}) .
$$

Moreover, following [9, Proposition 3] we also have

$$
\left(\mathcal{P}^{\pi^{*} \widehat{\mathcal{F}}}, \tilde{B}\right)_{q}=\left(\mathcal{P}^{\widehat{\mathcal{F}}}, B\right)_{p}-\left(\nu_{p}(\widehat{\mathcal{F}})+1-\epsilon(\widehat{\mathcal{F}})\right) \nu_{p}(B)+\nu_{q}(\tilde{B}) .
$$

Combining (4.1), (4.2) and Proposition 3.3 yields

$$
\begin{aligned}
\Delta_{q}\left(\pi^{*} \widehat{\mathcal{F}}, \tilde{B}\right) & =\Delta_{p}(\widehat{\mathcal{F}}, B)-\left(\nu_{p}(\widehat{\mathcal{F}})+1-\nu_{p}\left(\hat{F}_{p}\right)\right) \nu_{p}(B) \\
& =\Delta_{p}(\widehat{\mathcal{F}}, B)-\tau(\widehat{\mathcal{F}}) \nu_{p}(B) .
\end{aligned}
$$

The final statement follows from $\tau(\widehat{\mathcal{F}}) \geq 0$, this number vanishing if and only if $\widehat{\mathcal{F}}$ is a foliation of second type. 
Proposition 4.6. Let $\widehat{\mathcal{F}}$ be a germ of formal foliation at $p \in S$ having $\hat{F}$ as a balanced equation of separatrices. If $B$ is an irreducible component of $(\hat{F})_{0}$, then

$$
\Delta_{p}(\widehat{\mathcal{F}}, B) \geq 0
$$

Proof. The proof goes by induction on the length of the reduction process of $\widehat{\mathcal{F}}$. The inductive step is an obvious consequence of Proposition 4.5. The initialization in its turn follows from the three cases in Example 4.4.

As a consequence of the above, we obtain that the polar excess somehow gives a measure the existence of saddle-nodes in the desingularization of a foliation. This is the content of

THEOREM A. Let $\widehat{\mathcal{F}}$ be a germ of singular foliation at $p$ in a surface $S$ and $\hat{F}$ be a balanced equation for its separatrices. Then $\widehat{\mathcal{F}}$ is a generalized curve if and only if

$$
\Delta_{p}\left(\widehat{\mathcal{F}},(\hat{F})_{0}\right)=0 .
$$

Proof. Suppose first that $\widehat{\mathcal{F}}$ is a generalized curve. Then $\widehat{\mathcal{F}}$ is in particular a foliation of second type. For a fixed branch $B \subset(\hat{F})_{0}$, Proposition 4.5 asserts that the polar excess $\Delta$ is invariant under blowing-ups. Thus, it suffices to follow the transforms of $B$ along the reduction of singularities of $\widehat{\mathcal{F}}$. Now, from Example $4.4, \Delta_{p}(\widehat{\mathcal{F}}, B)>0$ if and only if $B$ is transformed into the weak separatrix of a saddle-node. We are however in the generalized curve case and we must have $\Delta_{p}(\widehat{\mathcal{F}}, B)=0$. We finally conclude that $\Delta_{p}\left(\widehat{\mathcal{F}},(\hat{F})_{0}\right)=0$ by summing up $\Delta_{p}(\widehat{\mathcal{F}}, B)$ for all branches $B \subset(\hat{F})_{0}$.

Reciprocally, note that $\Delta_{p}\left(\widehat{\mathcal{F}},(\hat{F})_{0}\right)=0$ implies that $\Delta_{p}(\widehat{\mathcal{F}}, B)=0$ for every branch $B \subset(\hat{F})_{0}$. Since $(\hat{F})_{0}$ is non-empty, Proposition 4.5 ensures that $\tau(\widehat{\mathcal{F}})=0$ and thus $\widehat{\mathcal{F}}$ is a foliation of second type. This means that all possible saddle-nodes in the reduction of $\widehat{\mathcal{F}}$ are well-oriented. On the other hand, the weak separatrix of a well-oriented saddlenode would contribute positively to the polar excess of $\widehat{\mathcal{F}}$, as shown in Example 4.4. This leads to the conclusion that saddle-nodes do not exist at all and, by definition, $\widehat{\mathcal{F}}$ is a generalized curve at $p$.

\section{Polar excess and the $G S V$-index.}

The $G S V$-index was defined by Gómez-Mont, Seade and Verjovsky in [19] for a holomorphic vector field on an analytic hypersurface $V$ at $\left(\mathbb{C}^{n}, 0\right)$ having isolated singularity. It is the Poincaré-Hopf index of a differentiable vector field obtained by isotopically displacing the original vector field over the Milnor fiber of $V$. The formulation below, for foliations at $\left(\mathbb{C}^{2}, 0\right)$ having an invariant curve, was introduced by Brunella in [4].

Definition 5.1. Let $\mathcal{F}$ be an analytic foliation at $\left(\mathbb{C}^{2}, 0\right)$ and $C$ be the union of some analytic separatrices of $\mathcal{F}$. If $\omega$ is a 1 -form that induces $\mathcal{F}$ and $f=0$ is a reduced equation for $C$, then it is possible to write a decomposition

$$
g \omega=k \mathrm{~d} f+f \eta,
$$


where $\eta$ is a 1 -form and $g, k \in \mathbb{C}\{x, y\}$ with $g$ and $f$ relatively prime. The $G S V$-index of $\mathcal{F}$ with respect to $C$ at $\left(\mathbb{C}^{2}, 0\right)$ is defined by

$$
G S V_{0}^{\mathrm{c}}(\mathcal{F}, C)=\frac{1}{2 \pi i} \int_{\partial C} \frac{g}{k} \mathrm{~d}\left(\frac{k}{g}\right) .
$$

Here $\partial C$ is the intersection $C \cap S_{\epsilon}^{3}$, where $S_{\epsilon}^{3}$ is a small sphere centered at $0 \in \mathbb{C}^{2}$, oriented as the boundary of $C \cap B_{\epsilon}^{4}$, for a ball $B_{\epsilon}^{4}$ such that $S_{\epsilon}^{3}=\partial B_{\epsilon}^{4}$.

The superscript "c" in $G S V_{0}^{\mathrm{c}}(\mathcal{F}, C)$ stresses the fact that this definition works in the convergent category. Nevertheless, we can extended the $G S V$-index to formal foliations using the following:

DEFINITION 5.2. Let $\widehat{\mathcal{F}}$ be a formal foliation at $\left(\mathbb{C}^{2}, 0\right)$ and $\hat{C}$ be a germ of separatrix of $\mathcal{F}$. If $\hat{\omega}$ is a 1 -form inducing $\widehat{\mathcal{F}}$ and $f=0$ is a reduced equation for $\hat{C}$, then, as in the convergent case, it is possible to write a decomposition

$$
g \hat{\omega}=k \mathrm{~d} f+f \hat{\eta}
$$

where $\hat{\eta}$ is a formal 1 -form and $g, k \in \mathbb{C}[[x, y]]$ with $g$ and $f$ relatively prime. Now, if $\gamma$ is a Puiseux parametrization of $\hat{C}$, we define

$$
G S V_{0}(\widehat{\mathcal{F}}, \hat{C})=\operatorname{ord}_{t=0} \frac{k}{g} \circ \gamma
$$

If $\hat{C}=\hat{C}_{0} \cup \hat{C}_{1}$ is the union of two disjoint sets of separatrices, then we define the $G S V$-index inductively by the formula

$$
G S V_{0}(\widehat{\mathcal{F}}, \hat{C})=G S V_{0}\left(\widehat{\mathcal{F}}, \hat{C}_{0}\right)+G S V_{0}\left(\widehat{\mathcal{F}}, \hat{C}_{1}\right)-2\left(\hat{C}_{0}, \hat{C}_{1}\right)_{0}
$$

where $\left(\hat{C}_{0}, \hat{C}_{1}\right)_{0}$ stands for the intersection number at $0 \in \mathbb{C}^{2}$.

The relation (5.1) simply follows the relation satisfied in the convergent category as shown in [4]. Thus, the following lemma is straightforward and justifies a posteriori the definition.

LEMma 5.3. Let $\mathcal{F}$ be a germ of analytic foliation and let $C$ be a union of convergent separatrices. Then

$$
G S V_{0}(\mathcal{F}, C)=G S V_{0}^{\mathrm{c}}(\mathcal{F}, C) .
$$

Next, we establish a link between the $G S V$-index and the relative polar excess.

Proposition 5.4. Let $\widehat{\mathcal{F}}$ be a germ of singular foliation at $p \in \mathbb{C}^{2}$ and $\hat{F}$ be a balanced equation of separatrices. If $B \subset(\hat{F})_{0}$ is irreducible then

$$
\Delta_{p}^{\mathrm{rel}}(\widehat{\mathcal{F}}, B)=G S V_{p}(\widehat{\mathcal{F}}, B)+\left((\hat{F})_{\infty}, B\right)_{p}
$$

Proof. Let us write $\omega=P \mathrm{~d} x+Q \mathrm{~d} y, \hat{F}=G / H$ and consider $f$ a reduced equation 
of $B$. The decomposition $g \omega=k \mathrm{~d} f+f \eta$ gives us

$$
a P+b Q=\frac{k}{g} \frac{\left(a \partial_{x} f+b \partial_{y} f\right)}{H} H+\frac{f}{g}\left(a \eta_{x}+b \eta_{y}\right),
$$

where $\eta=\eta_{x} \mathrm{~d} x+\eta_{y} \mathrm{~d} y$. Let $\gamma$ be a Puiseux parametrization of $B$. Since $f$ and $g$ are relatively prime, $g \circ \gamma$ is not identically zero. Moreover, by definition, $f \circ \gamma=0$. Hence, for a fixed $(a: b) \in \mathbb{P}_{\mathbb{C}}^{1}$, we obtain

$$
\underbrace{\operatorname{ord}_{t=0}(a P+b Q) \circ \gamma}_{\left(\mathcal{P}_{(a: b)}^{\hat{\mathcal{F}}}, B\right)_{p}}=\underbrace{\operatorname{ord}_{t=0} \frac{k}{g} \circ \gamma}_{G S V_{p}(\widehat{\mathcal{F}}, B)}+\underbrace{\operatorname{ord}_{t=0} \frac{\left(a \partial_{x} f+b \partial_{y} f\right)}{H}}_{\left(\mathcal{P}_{(a: b)}^{\mathrm{d}(f / H)}, B\right)_{p}}+\underbrace{\operatorname{ord}_{t=0} H \circ \gamma}_{\left((\hat{F})_{\infty}, B\right)_{p}},
$$

which, after taking generic $(a: b)$, gives the proposition.

Lemma 5.5. Let $B_{1}$ and $B_{2}$ be two branches of $(\hat{F})_{0}$. Then

$$
\Delta_{p}^{\mathrm{rel}}\left(\widehat{\mathcal{F}}, B_{1} \cup B_{2}\right)=\Delta_{p}^{\mathrm{rel}}\left(\widehat{\mathcal{F}}, B_{1}\right)+\Delta_{p}^{\mathrm{rel}}\left(\widehat{\mathcal{F}}, B_{2}\right)-2\left(B_{1}, B_{2}\right)_{p} .
$$

Proof. Let $f$ and $g$ be respectively the equations of $B_{1}$ and $B_{2}$. Let $\gamma$ be a Puiseux parametrization of $B_{1}$. For $(a: b) \in \mathbb{P}_{\mathbb{C}}^{1}$, we have

$$
\begin{aligned}
\left(\mathcal{P}_{(a: b)}^{d(f g / H)}, B_{1}\right)_{p} & =\operatorname{ord}_{t=0}\left(\frac{a \partial_{x}(f g)+b \partial_{y}(f g)}{H}\right) \circ \gamma \\
& =\operatorname{ord}_{t=0}\left(\frac{g\left(a \partial_{x} f+b \partial_{y} f\right)+f\left(a \partial_{x} g+b \partial_{y} g\right)}{H}\right) \circ \gamma \\
& =\operatorname{ord}_{t=0}(g \circ \gamma)+\operatorname{ord}_{t=0}\left(\frac{a \partial_{x} f+b \partial_{y} f}{H}\right) \circ \gamma \\
& =\left(B_{1}, B_{2}\right)_{p}+\left(\mathcal{P}_{(a: b)}^{d(f / H)}, B_{1}\right)_{p} .
\end{aligned}
$$

Since, by symmetry, the same holds for $B_{2}$, we get

$$
\begin{aligned}
\Delta_{p}^{\mathrm{rel}}\left(\widehat{\mathcal{F}}, B_{1} \cup B_{2}\right)= & \left(\mathcal{P}_{(a: b)}^{\widehat{\mathcal{F}}}, B_{1} \cup B_{2}\right)_{p}-\left(\mathcal{P}_{(a: b)}^{\mathrm{d}(f g / H)}, B_{1} \cup B_{2}\right)_{p} \\
= & \left(\mathcal{P}_{(a: b)}^{\widehat{\mathcal{F}}}, B_{1}\right)_{p}+\left(\mathcal{P}_{(a: b)}^{\widehat{\mathcal{F}}}, B_{2}\right)_{p} \\
& -\left(\mathcal{P}_{(a: b)}^{\mathrm{d}(f g / H)}, B_{1}\right)_{p}-\left(\mathcal{P}_{(a: b)}^{\mathrm{d}(f g / H)}, B_{2}\right)_{p} \\
= & \left(\mathcal{P}_{(a: b)}^{\widehat{\mathcal{F}}}, B_{1}\right)_{p}-\left(\mathcal{P}_{(a: b)}^{d(f / H)}, B_{1}\right)_{p} \\
& +\left(\mathcal{P}_{(a: b)}^{\widehat{\mathcal{F}}}, B_{2}\right)_{p}-\left(\mathcal{P}_{(a: b)}^{d(g / H)}, B_{2}\right)_{p} \\
& -2\left(B_{1}, B_{1}\right)_{p} \\
= & \Delta_{p}^{\mathrm{rel}}\left(\widehat{\mathcal{F}}, B_{1}\right)+\Delta_{p}^{\mathrm{rel}}\left(\widehat{\mathcal{F}}, B_{2}\right)-2\left(B_{1}, B_{2}\right)_{p} .
\end{aligned}
$$

By simple induction on the number of irreducible components, we can extend Proposition 5.4, replacing $B_{1}$ and $B_{2}$ by two disjoint sets of separatrices. Moreover, since the $G S V$-index satisfies the very same adjunction formula, this result implies that 
Corollary 5.6. For any set of separatrices $C \subset(\hat{F})_{0}$, one has

$$
\Delta_{p}^{\mathrm{rel}}(\widehat{\mathcal{F}}, C)=G S V_{p}(\widehat{\mathcal{F}}, C)+\left((F)_{\infty}, C\right)_{p} .
$$

In order to make a link between the total polar excess of $\widehat{\mathcal{F}}$ and its $G S V$-index, we will start by providing a connection between the total and the relative polar excess.

Lemma 5.7. Let $C \subset(\hat{F})_{0}$ be a set of separatrices. Then

$$
\Delta_{p}(\widehat{\mathcal{F}}, C)=\Delta_{p}^{\mathrm{rel}}(\widehat{\mathcal{F}}, C)-\left(C,(\hat{F})_{0} \backslash C\right)_{p}
$$

Proof. We will present the proof for a branch of separatrix. The general case is a simple induction based upon Lemma 5.5. As usual, denote $\hat{F}=G / H=g_{1} \cdots g_{n} / H$ and let $\gamma$ be a Puiseux parametrization of the irreducible component $B=\left\{g_{1}=0\right\}$ of $(\hat{F})_{0}$. Let us denote by $g$ the product $g_{2} \cdots g_{n}$. Then, for $(a: b) \in \mathbb{P}_{\mathbb{C}}^{1}$, we have

$$
\begin{aligned}
\left(\mathcal{P}_{(a: b)}^{d(G / H)}, B\right)_{p} & =\operatorname{ord}_{t=0}\left(\frac{a \partial_{x} G+b \partial_{y} G}{H}\right) \circ \gamma \\
& =\operatorname{ord}_{t=0}\left(g\left(\frac{a \partial_{x} g_{1}+b \partial_{y} g_{1}}{H}\right)\right) \circ \gamma \\
& =\underbrace{\operatorname{ord}_{t=0} g \circ \gamma}_{\left(B,(F)_{0} \backslash B\right)}+\underbrace{\operatorname{ord}_{t=0}\left(\frac{a \partial_{x} g_{1}+b \partial_{y} g_{1}}{H}\right) \circ \gamma}_{\left(\mathcal{P}_{(a: b)}^{d\left(g_{1} / H\right)}, B\right)_{p}},
\end{aligned}
$$

which ensures the lemma.

Finally, the combination of Corollary 5.6 and Lemma 5.7 yields the next result, which expresses the $G S V$-index in terms of the polar excess index and the balanced equation of separatrices.

TheOREM B. Let $\widehat{\mathcal{F}}$ be a germ of singular foliation at $\left(\mathbb{C}^{2}, p\right)$. Let $\hat{F}$ be a balanced equation of separatrices and $C$ be a union of isolated separatrices of $\widehat{\mathcal{F}}$. Then

$$
G S V_{p}(\widehat{\mathcal{F}}, C)=\Delta_{p}(\widehat{\mathcal{F}}, C)+\left(C,(\hat{F})_{0} \backslash C\right)_{p}-\left(C,(\hat{F})_{\infty}\right)_{p} .
$$

In particular, if $\widehat{\mathcal{F}}$ is a generalized curve then

$$
G S V_{p}\left(\widehat{\mathcal{F}},(\hat{F})_{0}\right)=-\left((\hat{F})_{0},(\hat{F})_{\infty}\right)_{p}
$$

In the non-dicritical case, the $G S V$-index and the polar excess index coincide, as shown in $[\mathbf{9}]$ :

COROLlaRY 5.8. If $\widehat{\mathcal{F}}$ is non-dicritical and $C$ is its complete set of separatrices, then

$$
G S V_{p}(\widehat{\mathcal{F}}, C)=\Delta_{p}(\widehat{\mathcal{F}}, C)
$$




\section{The Poincaré problem for dicritical singularities.}

We now work with global foliations on $\mathbb{P}_{\mathbb{C}}^{2}$. Let $\mathcal{F}$ be an analytic foliation of degree $d$ having an invariant algebraic curve $S$ of degree $d_{0}$. In [3] and [4], the sum of the $G S V$-indices over $S$ is expressed as

$$
\left(d+2-d_{0}\right) d_{0}=\sum_{p \in \operatorname{Sing}(\mathcal{F}) \cap S} G S V_{p}(\mathcal{F}, S) .
$$

Using the expression of the $G S V$-index in terms of polar excess given in Theorem B, we get a control of the degree of the invariant curve $d_{0}$ in terms of local invariants of the foliation. We have:

$$
\begin{aligned}
d_{0} & =d+2-\frac{1}{d_{0}} \sum_{p \in \operatorname{Sing}(\mathcal{F}) \cap S} G S V_{p}(\mathcal{F}, S) \\
& =d+2-\frac{1}{d_{0}} \sum_{p \in \operatorname{Sing}(\mathcal{F}) \cap S}\left(\Delta_{p}(\mathcal{F}, S)+\left(S,\left(\hat{F}_{p}\right)_{0} \backslash S\right)_{p}-\left(S,\left(\hat{F}_{p}\right)_{\infty}\right)_{p}\right),
\end{aligned}
$$

where, at each point $p \in \operatorname{Sing}(\mathcal{F}) \cap S$, we chose a balanced equation $\hat{F}_{p}$ adapted to to the local branches of $S$ at $p$. Using Proposition 4.6, we find

TheOREM C. Let $\mathcal{F}$ be an analytic foliation on $\mathbb{P}_{\mathbb{C}}^{2}$ of degreed having an invariant algebraic curve $S$ of degree $d_{0}$. Then

$$
d_{0} \leq d+2+\frac{1}{d_{0}} \sum_{p \in \operatorname{Sing}(\mathcal{F}) \cap S}\left[\left(S,\left(\hat{F}_{p}\right)_{\infty}\right)_{p}-\left(S,\left(\hat{F}_{p}\right)_{0} \backslash S\right)_{p}\right]
$$

where $\hat{F}_{p}$ is a balanced equation of separatrices adapted to to the local branches of $S$ at $p$. Besides, if all singularities of $\mathcal{F}$ over $S$ are generalized curves, then the equality holds.

This theorem allows us to recover the main result in $[\mathbf{1 0}]$ :

Corollary 6.1. If $\left(\hat{F}_{p}\right)_{\infty}=\emptyset$ for all $p \in \operatorname{Sing}(\mathcal{F}) \cap S$ then $d_{0} \leq d+2$. This happens in particular when all such points are non-dicritical.

\subsection{A dicritical foliation of degree 1 in $\mathbb{P}_{\mathbb{C}}^{2}$.}

As an example, let us consider the foliation of $\mathbb{P}_{\mathbb{C}}^{2}$ given by the meromorphic homogeneous first integral

$$
\omega=\mathrm{d}\left(\frac{x^{p} z^{q-p}}{y^{q}}\right)
$$

where $p<q$. This is a foliation of degree 1 on $\mathbb{P}_{\mathbb{C}}^{2}$ whose generic separatrix has degree $q$ and is given by $x^{p} y^{q-p}-c z^{q}=0$. Let us consider $S=\left\{x^{p} y^{q-p}-z^{q}=0\right\}$. The foliation has three singularities: two of them - denoted by $a$ and $b$ on the picture below-are dicritical, the other one is a saddle. 


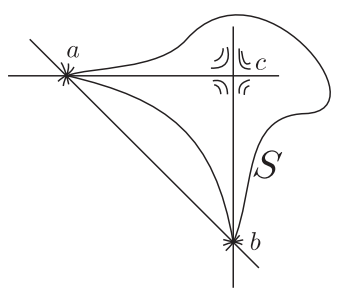

Figure 6.1. The foliation of $\mathbb{P}_{\mathbb{C}}^{2}$ given by $\omega=\mathrm{d}\left(x^{p} z^{q-p} / y^{q}\right)$.

In this situation, the sum in inequality (6.1) reduces to

$$
\left(S,\left(F_{a}\right)_{\infty}\right)_{a}-\left(S,\left(F_{a}\right)_{0} \backslash S\right)_{a}+\left(S,\left(F_{b}\right)_{\infty}\right)_{b}-\left(S,\left(F_{b}\right)_{0} \backslash S\right)_{b} .
$$

In a neighborhood of $a$, in the affine chart $z=1$, we can choose

$$
\hat{F}_{a}=\frac{x y\left(x^{p}-y^{q}\right)}{x^{p}-2 y^{q}}
$$

as a balanced equation. Thus

$$
\begin{aligned}
\left(S,\left(F_{a}\right)_{\infty}\right)_{a}-\left(S,\left(F_{a}\right)_{0} \backslash S\right)_{a} & =\left(x^{p}-y^{q}, x^{p}-2 y^{q}\right)_{0}-\left(x^{p}-y^{q}, x y\right)_{0} \\
& =p q-p-q .
\end{aligned}
$$

On the other hand, in a neighborhood of $b$ and in the affine chart $x=1, \hat{F}_{b}=$ $z y\left(z^{q-p}-y^{q}\right) /\left(z^{q-p}-2 y^{q}\right)$ is a balanced equation of separatrices. Thus

$$
\begin{aligned}
\left(S,\left(F_{b}\right)_{\infty}\right)_{b}-\left(S,\left(F_{b}\right)_{0} \backslash S\right)_{b} & =\left(z^{q-p}-y^{q}, z^{q-p}-2 y^{q}\right)_{0}-\left(z^{q-p}-y^{q}, z y\right)_{0} \\
& =q^{2}-q p-2 q+p .
\end{aligned}
$$

Finally, we get checked the equality

$$
\underbrace{d_{0}}_{=q}=\underbrace{d}_{=1}+2-\frac{1}{q}\left((p q-p-q)+\left(q^{2}-q p-2 q+p\right)\right) .
$$

\subsection{The pencil of Lins Neto.}

One of the most remarkable counterexamples for the Poincaré's problem is the pencil of Lins Neto (see [20]) defined by

$$
\begin{aligned}
\mathcal{P}_{\alpha} & =\omega+\alpha \eta \\
\omega & =\left(x^{3}-1\right) x \mathrm{~d} y+\left(y^{3}-1\right) y \mathrm{~d} x \\
\eta & =\left(x^{3}-1\right) y^{2} \mathrm{~d} y+\left(y^{3}-1\right) x^{2} \mathrm{~d} x .
\end{aligned}
$$

When $\alpha \in \mathbb{Q}(j)$ where $j=e^{2 i \pi / 3}$, the foliation $\mathcal{P}_{\alpha}$ of $\mathbb{P}_{\mathbb{C}}^{2}$ of degree 4 admits a meromorphic first integral whose degree cannot be bounded, although the singular locus and the local analytical type of the singularities do not depend on $\alpha$. Actually, when $\alpha \in \mathbb{Q}(j) \backslash\left\{1, j, j^{2}, \infty\right\}$, the singular locus consists of 21 points: 9 of them are non- 
degenerate singularities and the 12 remaining are radial singularities, given in local coordinates $(u, v)$ by $\mathrm{d}(u / v)=0$. These singularities are the locus of intersection of the 9 lines defined in homogeneous coordinates by

$$
\left(x^{3}-y^{3}\right)\left(x^{3}-z^{3}\right)\left(y^{3}-z^{3}\right)=0 .
$$

The group of automorphisms of $\mathcal{P}_{\alpha}$ is generated by the following five transformations:

$$
\begin{gathered}
{[x: y: z] \mapsto[y: x: z],[x: y: z] \mapsto[z: y: x],[x: y: z] \mapsto[x: z: y],} \\
{[x: y: z] \mapsto\left[j x: j^{2} y: z\right],[x: y: z] \mapsto\left[j^{2} x: j y: x\right] .}
\end{gathered}
$$

The action of this group on the radial singular points divides them in 4 classes of 3 points:

$$
\begin{aligned}
& \left\{s_{1}=[0: 0: 1],[0: 1: 0],[1: 0: 0]\right\}, \\
& \left\{s_{2}=[1: 1: 1],\left[j: j^{2}: 1\right],\left[j^{2}: j: 1\right]\right\}, \\
& \left\{s_{3}=[1: j: 1],[j: 1: 1],\left[j^{2}: j^{2}: 1\right]\right\}, \\
& \left\{s_{4}=\left[1: j^{2}: 1\right],[j: j: 1],\left[j^{2}: 1: 1\right]\right\} .
\end{aligned}
$$

Let us now consider $\alpha \in \mathbb{Q}(j) \backslash\left\{1, j, j^{2}, \infty\right\}$ and $S$ a generic invariant algebraic curve of $\mathcal{P}_{\alpha}$. The intersection of $S$ with the singular locus contains only radial singularities, because the set of algebraic invariant curves passing through the non-degenerated singularities is finite. Let us denote $N_{i}$ the number of times that $S$ goes through the singular point $s_{i}$. This number does not depend on the choice of $S$. In view of the description of the group of automorphisms of $\mathcal{P}_{\alpha}$, for any point $\tilde{s}_{i}$ in the same orbit of $s_{i}, S$ goes through $\tilde{s}_{i}$ also $N_{i}$ times. The following lemma is easy:

LEMma 6.2. Let $\mathcal{R}$ be the germ of radial foliation $x \mathrm{~d} y-y \mathrm{~d} x$ and $S$ be a union of $N$ invariant curves. Let $F$ be an adapted balanced equation. Then

$$
\left(S,(F)_{\infty}\right)_{0}-\left(S,(F)_{0} \backslash S\right)_{0}=N^{2}-2 N .
$$

Therefore, according to Theorem $\mathrm{C}$, we have

$$
\begin{aligned}
d_{0}^{2}-6 d_{0} & =\sum_{s \in \operatorname{Sing}(S)\left(\mathcal{P}_{\alpha}\right) \cap S}\left\{\left(S,\left(F_{s}\right)_{\infty}\right)_{s}-\left(S,\left(F_{s}\right)_{0} \backslash S\right)_{s}\right\} \\
& =3 \sum_{i=1}^{4}\left(N_{i}^{2}-2 N_{i}\right) .
\end{aligned}
$$

Thus, we find

$$
d_{0}=3+\sqrt{9+3 \sum_{i=1}^{4}\left(N_{i}^{2}-2 N_{i}\right)} .
$$

Now, in view of the description in [23], the numbers $N_{i}$ can be computed in the following way: let us write $\alpha=\alpha_{1} / \beta_{1}$, where $\alpha_{1}, \beta_{1} \in \mathbb{Z}[j]$ are relatively prime. Then 


$$
N_{1}=N\left(\alpha_{1}\right), N_{2}=N\left(\beta_{1}\right), N_{3}=N\left(\alpha_{1}-\beta_{1}\right) \text { and } N_{4}=N\left(\alpha_{1}+j \beta_{1}\right),
$$

where $N(a+j b)=a^{2}+b^{2}-a b$. Therefore, the radicand in equation (6.2) is

$$
\begin{aligned}
9+3 \sum_{i=1}^{4}\left(N_{i}^{2}-2 N_{i}\right)=9+3\left(N\left(\alpha_{1}\right)^{2}-2 N\left(\alpha_{1}\right)+N\left(\beta_{1}\right)^{2}-2 N\left(\beta_{1}\right)\right) \\
+3\left(N\left(\alpha_{1}-\beta_{1}\right)^{2}-2 N\left(\alpha_{1}-\beta_{1}\right)+N\left(\alpha_{1}+j \beta_{1}\right)^{2}-2 N\left(\alpha_{1}+j \beta_{1}\right)\right) .
\end{aligned}
$$

This gives

$$
d_{0}=N\left(\alpha_{1}\right)+N\left(\beta_{1}\right)+N\left(\alpha_{1}-\beta_{1}\right)+N\left(\alpha_{1}+j \beta_{1}\right),
$$

which is consistent with $[\mathbf{2 8}]$.

\section{Topologically bounded invariants of a singularity.}

The singularity $p x \mathrm{~d} y-q y \mathrm{~d} x=0$, where $p$ and $q$ are coprime non-negative numbers, can be desingularized by a blowing-up process following Euclid's algorithm applied to the couple $(p, q)$. The algebraic multiplicity of the non-isolated separatrix is $\min \{p, q\}$, whereas its Milnor number is 1 for any $p$ and $q$. Thus, neither the length of the whole reduction process of a foliation nor the algebraic multiplicity of a general invariant curve can be bounded by a function of the Milnor number. This remark brings us to introduce the following definition:

Definition 7.1. Let $\operatorname{Fol}\left(\mathbb{C}^{2}, 0\right)$ denote the set germs of singular foliations at $\left(\mathbb{C}^{2}, 0\right)$. A numerical datum in $\operatorname{Fol}\left(\mathbb{C}^{2}, 0\right)$ is a function $\mathfrak{N}: \operatorname{Fol}\left(\mathbb{C}^{2}, 0\right) \rightarrow \mathbb{Z}$. We say that the numerical datum $\mathfrak{N}$ is topologically bounded if there exists a function $\psi: \mathbb{N} \rightarrow \mathbb{N}$ such that, for any foliation $\mathcal{F}$,

$$
|\mathfrak{N}(\mathcal{F})| \leq \psi\left(\mu_{0}(\mathcal{F})\right),
$$

where $\mu_{0}(\mathcal{F})$ is the Milnor number of $\mathcal{F}$.

Notice that the sum of two topologically bounded numerical data is topologically bounded. The algebraic multiplicity of a foliation is topologically bounded, since

$$
\nu_{0}(\mathcal{F}) \frac{\left(\nu_{0}(\mathcal{F})+1\right)}{2} \leq \mu_{0}(\mathcal{F}) .
$$

Indeed, if $\mathcal{F} \in \operatorname{Fol}\left(\mathbb{C}^{2}, 0\right)$ is given by $\omega=a \mathrm{~d} x+b \mathrm{~d} y$ then

$$
\mu=\operatorname{dim}_{\mathbb{C}} \frac{\mathbb{C}\{x, y\}}{(a, b)}=\operatorname{dim}_{\mathbb{C}} \frac{\mathbb{C}\{x, y\}}{(x, y)^{\nu}} \times \frac{(x, y)^{\nu}}{(a, b)} \geq \operatorname{dim}_{\mathbb{C}} \frac{\mathbb{C}\{x, y\}}{(x, y)^{\nu}}=\frac{\nu(\nu+1)}{2},
$$

where $\mu=\mu_{0}(\mathcal{F})$ and $\nu=\nu_{0}(\mathcal{F})$. However, as illustrated by the above example, the length of the reduction process is not topologically bounded. In [15], Corral and Fernández proved the following: 
THEOREM 7.2. Let $\mathcal{F}$ be a germ of foliation at $\left(\mathbb{C}^{2}, 0\right)$ and $S$ be the union of its formal isolated separatrices. Then the algebraic multiplicity of $S$ and the length of its reduction process are topologically bounded.

In this section, we intend to extend this result to a wider class of invariant curves. This is the content of:

TheOREM D. Let $\mathcal{F}$ be a germ of foliation in $\mathbb{C}^{2}$. For a fixed $r \in \mathbb{N}$, let $S$ be formed by the union of the following curves:

(a) all formal isolated separatrices;

(b) one copy of non-isolated separatrix attached to each dicritical component of valence one;

(c) $r$ copies of non-isolated separatrices attached to each dicritical component of valence three or greater.

Then the algebraic multiplicity of $S$ and the length of its reduction process are topologically bounded.

\subsection{Topological boundedness for saddle-nodes singularities.}

In this section, we are going to prove the following lemma:

LEMMA 7.3. The following data, concerning singularities appearing along the reduction process, are topologically bounded:

(a) Milnor numbers;

(b) The number of saddle-node and their weak indices.

Proof. Let $E_{1}$ be a single blowing-up at the singular point $p$ and let $D_{1}=E_{1}^{-1}(p)$. The following formulas are classical (see $[\mathbf{2 1}]$ ):

$$
\mu_{p}(\mathcal{F})= \begin{cases}\nu_{p}^{2}(\mathcal{F})-\nu_{p}(\mathcal{F})-1+\sum_{p^{\prime} \in D_{1}} \mu_{p^{\prime}}\left(E_{1}^{*} \mathcal{F}\right) & \text { if } E_{1} \text { is non-dicritical } \\ \nu_{p}^{2}(\mathcal{F})+\nu_{p}(\mathcal{F})-1+\sum_{p^{\prime} \in D_{1}} \mu_{p^{\prime}}\left(E_{1}^{*} \mathcal{F}\right) & \text { else. }\end{cases}
$$

Milnor numbers usually decrease with blowing-ups. Suppose however that, in the reduction process of $\mathcal{F}$, there is a blowing-up for which this fails. We focus at the first moment this happens, denoting by $E$ the sequence of blowing-ups done so far and by $c$ the singular point of $E^{*} \mathcal{F}$ that, once blown-up, produces a singularity with higher or equal Milnor number. According to formulas (7.1), such blowing-up has to be non-dicritical and the algebraic multiplicity must satisfy

$$
\nu_{c}\left(E^{*} \mathcal{F}\right)=1
$$

$E$ is an intermediate step in the minimal reduction process, thus $c$ is not a reduced singularity. Thus, looking at all possible cases, we are limited to the following two: 
(i) $\left(E^{*} \mathcal{F}\right)_{c}$ is given by $\omega=p x \mathrm{~d} y-q x \mathrm{~d} x+\cdots$. In that case, all Milnor numbers in the reduction process are 1 or less.

(ii) $\left(E^{*} \mathcal{F}\right)_{c}$ is nilpotent and, following [14], is given locally by

$$
\mathrm{d}\left(y^{2}+x^{n}\right)+x^{p} U(x) \mathrm{d} y .
$$

Here $\mu_{c}\left(E^{*} \mathcal{F}\right)=n-1$. One blowing-up yields a singular point with algebraic multiplicity 2 and Milnor number $n$. After this point, Milnor numbers decrease. Anyhow, along the blowing-up process starting at $c$, they cannnot exceed $n$.

We therefore conclude that, along the reduction process of $\mathcal{F}$, Milnor numbers cannot exceed $\mu_{0}(\mathcal{F})+1$.

For the second part of the lemma, consider the formal normal form of a saddle-node singularity:

$$
x^{p+1} \mathrm{~d} y+y\left(p-\lambda x^{p}\right) \mathrm{d} x, p \geq 1 .
$$

Its Milnor number and its weak index are both $p+1$, which gives the topological boundedness for the latter. Now, let $N$ be the number of saddle-nodes in the reduction of $\mathcal{F}$. We are going to prove by induction on the length of the reduction process that

$$
N \leq \mu_{0}(\mathcal{F})
$$

If the length is 0 , then the singularity is reduced. Thus $N \leq 1 \leq \mu_{0}(\mathcal{F})$. Let $k \geq 1$ and suppose that the proposition is proved for foliations with reduction process of length smaller than $k$. Consider a foliation $\mathcal{F}$ with reduction process of length $k$. Blow-up $\mathcal{F}$ once. The resulting foliation $E_{1}^{*} \mathcal{F}$ has a finite number of singularities $c_{1}, \ldots, c_{p}$. For any $i=1, \ldots, p$, the local foliation $\left(E_{1}^{*} \mathcal{F}\right)_{c_{i}}$ has a reduction process of length less than $k$, giving rise to $n_{i}$ saddle-node singularities. Evidently $\sum_{i=1}^{p} n_{i}=N$. Besides, by induction, $n_{i} \leq \mu_{c_{i}}\left(E_{1}^{*} \mathcal{F}\right)$ for $i=1, \ldots, p$. We have:

(i) If $\nu_{0}(\mathcal{F}) \geq 2$ then, according to $(7.1)$,

$$
\mu_{0}(\mathcal{F}) \geq \sum_{i=1}^{p} \mu_{c_{i}}\left(E_{1}^{*} \mathcal{F}\right) \geq \sum_{i=1}^{p} n_{i}=N .
$$

(ii) If $\nu_{0}(\mathcal{F})=1$, then either $\mathcal{F}$ is of the form $\omega=p x \mathrm{~d} y-q x \mathrm{~d} x+\cdots$, and no saddlenodes appear in its reduction, or $\mathcal{F}$ is nilpotent. In the second case, $\mu_{0}(\mathcal{F}) \geq 2$ and $N \leq 1$ and the inequality also holds.

7.2. Dicritical components of valences $\mathrm{v}(D)=1$ and $\mathrm{v}(D) \geq 3$.

We have the following properties concerning dicritical components of valence one:

LEMMA 7.4. The number and the multiplicities of the dicritical components of valence one are topologically bounded.

Proof. We perform a blowing-up process $E$ in order to reduce all formal isolated separatrices of $\mathcal{F}$. According to Theorem 7.2, the number of blowing-ups composing $E$ 
is topologically bounded. Then, the dicritical components of valence 1 in the exceptional divisor of $E$ have topologically bounded multiplicities.

The remaining dicritical singularities of $E^{*} \mathcal{F}$ are of special kind: they are crossed by at most one isolated separatrix of $\mathcal{F}$, which are smooth and transversal to the divisor $\mathcal{D}=E^{-1}(0)$. If $c \in \mathcal{D}$ is a singularity of $E^{*} \mathcal{F}$, then the reduction process of $\left(E^{*} \mathcal{F}\right)_{c}$ cannot have dicritical components of valence three or greater. To see this, we use the fact that each invariant connected component of the reduction divisor carries an isolated separatrix, as done in the proof of Lemma 3.4. If such a dicritical component existed, then we would find isolated separatrices outside $\mathcal{D}$, one or two, respectively if $c$ is a corner or a non-corner point of $\mathcal{D}$. This is a contradiction, since all isolated separatrices of $\mathcal{F}$ are reduced.

The reduction process of $\left(E^{*} \mathcal{F}\right)_{c}$ thus contains only dicritical components of valences 1 or 2 and at most one isolated separatrix of $\mathcal{F}$ attached to some invariant component of multiplicity one. The expression for the algebraic multiplicity given by Proposition 3.3 and Formula (3.2) lead to

$$
\nu_{c}\left(\left(E^{*} \mathcal{F}\right)_{c}\right)=\sum_{D \text { dicritical, } v(D)=1} \rho(D)+(\cdots),
$$

where the dots is an expression that depends only on the saddle-node singularities and their weak indices. Since these data are topologically bounded, this formula ensures that the number and multiplicities of dicritical components of valence one are topologically bounded.

In what concerns dicritical components of higher valences, we achieve the following:

COROllary 7.5 (of the proof). All dicritical components of valence three or greater appear after a topologically bounded number of blowing-ups. Besides, their number, multiplicities and valences are also topologically bounded.

Proof. All dicritical components of valence at least three appear as soon as all isolated separatrices are desingularized. It then follows from Theorem 7.2 that a topologically bounded number of blowing-ups create them. This is enough to bound their number and multiplicities. For their valences, it suffices to notice that, for a dicritical component $D, \mathrm{v}(D) \leq \iota(D)+2$, where $\iota(D)$ is the number of isolated separatrices originating on $D$.

\subsection{Proof of Theorem D.}

Proof of Theorem D. We observe that, in formulas $(7.1)$, if $\nu_{p}(\mathcal{F})>2$ then both second degree expressions in $\nu_{p}(\mathcal{F})$ provide strictly positive integers. Let us then apply these formulas inductively along the reduction process of $\mathcal{F}$ as long as the blownup singularities have algebraic multiplicities at least 2 . In doing so we are led to an expression of the form

$$
\mu(\mathcal{F})=(\cdots)+\sum_{c \in \mathcal{D}} \mu_{c}\left(E^{*} \mathcal{F}\right)
$$


where $E$ is the blowing-up process and $\mathcal{D}=D^{-1}(0)$ is the exceptional divisor. The expression represented by $(\cdots)$ is a positive integer, greater than or equal to the number of blowing-ups in $E$. In particular, this number is at most $\mu(\mathcal{F})$ and, thus, topologically bounded. Let $c \in \mathcal{D}$ and $S_{c}$ be the local component of the strict transform of $S$ by $E$. The multiplicity $\nu_{c}\left(E^{*} \mathcal{F}\right)$ is equal to 0 or 1 . Thus, the foliation $\left(E^{*} \mathcal{F}\right)_{c}$ belongs to the following list:

(i) A regular foliation.

(ii) A simple singularity with two eigenvalues whose quotient is not a positive rational number, $\omega=\lambda_{1} x \mathrm{~d} y-\lambda_{2} y \mathrm{~d} x+\cdots$, with $\lambda_{1} / \lambda_{2} \notin \mathbb{Q}^{+}$.

(iii) A Dulac singularity, $\omega=x \mathrm{~d} y-n y \mathrm{~d} x+\cdots$, with $n \in \mathbb{N}^{*}$. The normal form is given by $\left(x+a y^{n}\right) \mathrm{d} y-n y \mathrm{~d} x$. The foliation is dicritical if and only if $a=0$. If $a \neq 0$ then it has only one regular separatrix.

(iv) A non-degenerated dicritical singularity, $\omega=p x \mathrm{~d} y-q y \mathrm{~d} x+\cdots$, with $p, q \in \mathbb{N}^{*}$ and $p / q \notin \mathbb{N} \cup(1 / \mathbb{N})$.

(v) A nilpotent singularity, $\omega=y \mathrm{~d} y+\cdots$.

If the singularity of $\left(E^{*} \mathcal{F}\right)_{c}$ provides only isolated separatrices in $S_{c}$ then, according to Theorem 7.2, the number of blowing-ups in their reduction process is topologically bounded. From previous lemmas and results about simple, Dulac, resonnant and nilpotent singularities in $[\mathbf{1}],[\mathbf{1 4}],[\mathbf{2 4}],[\mathbf{2 5}]$, the local curve $S_{c}$ possibly contains a dicritical separatrix only in the following three cases:
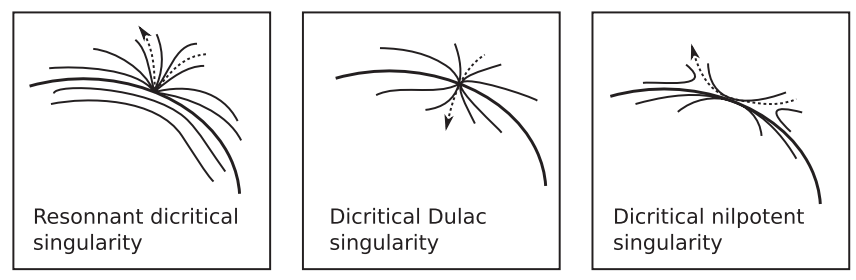

Their analysis depends on whether or not $c$ is a corner of $\mathcal{D}$.

First case $: c \in \mathcal{D}$ is a non-corner point. Denoting by $D$ the local irreducible component of $\mathcal{D}$ at $c$, we have the following possibilities:

(i) $\left(E^{*} \mathcal{F}\right)_{c}$ is a resonant dicritical singularity of the form $p x \mathrm{~d} y-q y \mathrm{~d} x$ with $p / q \notin$ $\mathbb{N} \cup(1 / \mathbb{N})$. Then all leaves are singular, except those given by $x=0$ and $y=0$. Thus, locally $D$ is defined by $x=0$, by $y=0$ or is transverse to the foliation. In all cases, the reduction process will produce a unique dicritical component of valence 2 . So the local trace of $S_{c}$ reduces to $x=0$ or to $y=0$, which are isolated separatrices.

(ii) $\left(E^{*} \mathcal{F}\right)_{c}$ is a dicritical Dulac singularity of the form $x \mathrm{~d} y-n y \mathrm{~d} x$. Then all leaves are smooth, given by $x=0$ or by $y=\alpha x^{n}$, with $\alpha \in \mathbb{C}$. If $D$ is locally given by 
$x=0$, then the local trace of $S_{c}$ is some leaf $y=\alpha x^{n}$, which is smooth transverse to $\mathcal{D}$ and thus reduced. If $\mathcal{D}$ is of the form $y=\alpha x^{n}$, then either the local trace of $S_{c}$ is $x=0$, hence reduced, or it is given by $y=\beta x^{n}$ with $\beta \neq \alpha$. In the latter case, the reduction process provides a dicritical component of valence 2 , which is not allowed in the definition of $S$. Suppose finally that $D$ is not invariant. If it is transverse to every leaf or tangent to $x=0$, then the local trace of $S$ is written as $x\left(y-\alpha x^{n}\right)=0$ for some $\alpha$ and is reduced after one blowing-up. If it is tangent to $y=0$, then two cases can occur: if $D$ is tangent to $y=0$ with an order $n$ or greater, then the reduction process of $\left(E^{*} \mathcal{F}\right)_{c}$ produces a dicritical component of valence 2. If $D$ is tangent to $y=0$ with an order $p<n$, then the dicritical component $D_{0}$ appearing in the reduction process of $\left(E^{*} \mathcal{F}\right)_{c}$ is of valence 1 . However, since the multiplicity is an additive function, we have

$$
\rho\left(D_{0}\right)=p \rho(D) \geq p .
$$

Lemma 7.4 ensures that $\rho\left(D_{0}\right)$ is topologically bounded and so is the integer $p$. Now, after $p+1$ blowing-ups the curves $S_{c}=\left\{y=\alpha x^{n}\right\}$ and $D$ are separated. So, $S_{c}$ becomes reduced after a topologically bounded number of blowing-ups.

(iii) $\left(E^{*} \mathcal{F}\right)_{c}$ is a dicritical nilpotent singularity. Then after a topologically bounded number of blowing-ups, we are led to a resonant or Dulac dicritical singularity $c^{\prime}$. Indeed, according to [24], [25], in this situation, $2 p=n=\mu\left(\left(E^{*} \mathcal{F}\right)_{c}\right)$, so the integer $p$ is topologically bounded. If $c^{\prime}$ is a regular point of the exceptional divisor, then we are in situations (i) or (ii) above. The case where $c^{\prime}$ is a corner will be treated below.

Second case $c \in \mathcal{D}$ is at a corner. Now we denote locally $(\mathcal{D})_{c}=D_{1} \cup D_{2}$. The following subcases occur:

(i) $\left(E^{*} \mathcal{F}\right)_{c}$ is a resonant dicritical singularity. The proof goes as in First case, (i). All leaves except $x=0$ and $y=0$ are singular and the reduction process produces a dicritical component of valence 2 . Therefore, $S_{c}$ coincides with one of the isolated separatrices $x=0$ or $y=0$.

(ii) $\left(E^{*} \mathcal{F}\right)_{c}$ is a dicritical Dulac singularity. Suppose first that both local components of $\mathcal{D}$ are invariant. Then necessarily $(\mathcal{D})_{c}$ has an equation of the form $x\left(y-\alpha x^{n}\right)$ for some $\alpha \in \mathbb{C}$. The reduction process produces a dicritical component of valence 2 and $S_{c}$ contains no dicritical components.

Suppose now that only one local component of $(\mathcal{D})_{c}$, say $D_{1}$, is invariant. If $D_{1}=$ $\{x=0\}$ and $D_{2}$ is transverse to $y=0$, then $S_{c}$ is of the form $y=\alpha x^{n}$ for some $\alpha \neq 0$. In this situation, one more blowing-up reduces $S_{c}$. Suppose next that $D_{2}$ is tangent to $y=0$. Then two cases can occur: if $D_{2}$ is tangent to $y=0$ with order at least $n$, then the reduction process of $\left(E^{*} \mathcal{F}\right)_{c}$ produces a dicritical component of valence 2. On the other hand, if $D_{2}$ is tangent to $y=0$ with order $p<n$, then the dicritical component $D_{0}$ appearing in the reduction of $\left(E^{*} \mathcal{F}\right)_{c}$ has valence 1 . The multiplicity of $D_{0}$ is written 


$$
\rho\left(D_{0}\right)=\rho\left(D_{1}\right)+p \rho\left(D_{2}\right) \geq p .
$$

Lemma 7.4 assures that $\rho\left(D_{0}\right)$ is topologically bounded and so is the integer $p$. Now, after $p+1$ blowing-ups, the curve $S_{c}=\left\{y=\alpha x^{n}\right\}$ and $D_{2}$ are separated. So, $S_{c}$ is reduced after a topologically bounded number of blowing-ups. If $D_{1}=$ $\left\{y=\alpha x^{n}\right\}$ for some $\alpha \in \mathbb{C}$, then $D_{2}$ is transverse to $y=0$. Thus, the reduction process produces a dicritical component of valence 2. Hence $S_{c}$ is the isolated separatrix $x=0$.

Suppose that both components $D_{1}$ and $D_{2}$ are non-invariant. As before, we have the following alternatives: if neither $D_{1}$ nor $D_{2}$ is tangent to $y=0$ then one blowing-up is enough to reduce $S_{c}$. If one of the components is tangent to $y=0$, then either the order of tangency is greater than or equal to $n$ and a dicritical component of valence 2 appears, or it is smaller than $n$ and, then, topologically bounded.

(iii) $\left(E^{*} \mathcal{F}\right)_{c}$ is a dicritical nilpotent singularity. Then after a topologically bounded number of blowing-ups, we are led either to a resonant or to a Dulac dicritical singularity. These cases were already treated.

This ends the proof of Theorem D.

\subsection{Consequences to the Poincaré problem.}

Theorems $\mathrm{C}$ and $\mathrm{D}$ allow us to give an answer to the Poincaré problem in the dicritical case which generalizes the one in $[\mathbf{1 5}]$ :

Theorem E. For every $d, r \in \mathbb{N}$ there exists $N=N(d, r) \in \mathbb{N}$ with the following property: if $\mathcal{F}$ is an analytic foliation on $\mathbb{P}_{\mathbb{C}}^{2}$ of degree $d$ having an invariant algebraic curve $S$ of degree $d_{0}$ such that, for every $p \in \operatorname{Sing}(\mathcal{F}) \cap S$, the local component $(S)_{p}$ is composed by:

(a) any number of isolated separatrices;

(b) at most one separatrix attached to each dicritical component of valence one;

(c) no branches of separatrices attached to dicritical components of valence two;

(d) at most $r$ branches of separatrices attached to each dicritical component of valence three or greater;

(e) if $p$ is a radial singularity, at most $r$ branches of separatrices attached to the dicritical component of valence zero.

Then $d_{0} \leq N$.

Proof. We start by remarking that Bézout's Theorem for foliations,

$$
\sum_{p \in \operatorname{Sing}(\mathcal{F})} \mu_{p}(\mathcal{F})=d^{2}+d+1,
$$


gives a bound for the number of points $p \in \operatorname{Sing}(\mathcal{F})$ as well as for each $\mu_{p}(\mathcal{F})$ in terms of $d$. Besides, Theorem D gives that the reduction process of $(S)_{p}$ has a topologically bounded length. All we have to do is to find a topological bound to the term $\left(S,\left(\hat{F}_{p}\right)_{\infty}\right)_{p}$ in Theorem $\mathrm{C}$, where $\hat{F}_{p}$ is a balanced equation adapted to the invariant curve $S$ at $p$. Hypotheses (b) and (c) on the structure of $(S)_{p}$ imply that all branches in $\left(\hat{F}_{p}\right)_{\infty}$ are associated to dicritical components of valence $v(D) \geq 3$. By Lemma 7.5, these components arise after a topologically bounded number of blowing-ups, their number and their valences are also topologically bounded. This is enough to conclude that the number of branches and the length of the reduction process of $\left(\hat{F}_{p}\right)_{\infty}$ are both topologically bounded. In the same way, $S_{p} \cup\left(\hat{F}_{p}\right)_{\infty}$ has a reduction process of topologically bounded length. This is enough to bound $\left(S,\left(\hat{F}_{p}\right)_{\infty}\right)_{p}$ in terms of $\mu_{p}(\mathcal{F})$, and thus in terms of $d$.

In a sense, Theorem E provides the ultimate criterion to obtain a bound of the degree of an invariant curve from local topological data associated to the singularities of a foliation. Indeed, the foliations described in Sections 6.1 and 6.2 violate the conclusion of Theorem E. They appear to be prototypes of foliations from which one can construct exemples of families of foliations having algebraic invariant curves that do not satisfy conditions (b), (c), (d) or (e).

\section{Topological invariance of the algebraic multiplicity.}

Let $\mathcal{F}_{1}, \mathcal{F}_{2} \in \operatorname{Fol}\left(\mathbb{C}^{2}, 0\right)$ be two local analytic foliations. We say that a germ of homeomorphism $\Phi:\left(\mathbb{C}^{2}, 0\right) \rightarrow\left(\mathbb{C}^{2}, 0\right)$ is a topological equivalence between $\mathcal{F}_{1}$ and $\mathcal{F}_{2}$ if $\Phi$ takes leaves of $\mathcal{F}_{1}$ into leaves of $\mathcal{F}_{2}$. The Milnor number (see [5]) of a foliation and the $G S V$-index (see [19]) are well known topological invariants. On the other hand, the topological invariance of the algebraic multiplicity is so far not known. This is true when $\mathcal{F}$ is either a generalized curve foliation (by [5]) - in which case all separatrices are convergent - or a non-dicritical second type foliation with only convergent separatrices (by $[\mathbf{2 2}])$.

Let $\Phi:\left(\mathbb{C}^{2}, 0\right) \rightarrow\left(\mathbb{C}^{2}, 0\right)$ be a topological equivalence between foliations $\mathcal{F}_{1}, \mathcal{F}_{2} \in$ $\operatorname{Fol}\left(\mathbb{C}^{2}, 0\right)$. It is clear that if $S$ is a convergent separatrix of $\mathcal{F}_{1}$ then $\Phi_{*} S:=\Phi(S)$ is a convergent separatrix for $\mathcal{F}_{2}$. Actually, $\Phi(S)$ is an analytic curve as a consequence of Remmert-Stein Theorem, since $\overline{\Phi(S \backslash\{0\})}=\Phi(S \backslash\{0\}) \cup\{0\}$. We recall that nonconvergent separatrices appear as the weak separatrices of non-tangent saddle-nodes and thus are all isolated separatrices. On the other hand, dicritical separatrices converge.

We start by a remark: a topological equivalence respects the "dicritical structure" of the desingularization. This means that the same combinatory of blowing-ups that desingularizes the dicritical separatrices of $\mathcal{F}_{1}$, when applied to $\mathcal{F}_{2}$, will produce dicritical components at exactly the same positions. This is a consequence of Zariski's Equidesingularization Theorem for curves (see for instance $[\mathbf{2}]$ ). Actually, if $D$ is a dicritical component of the desingularization of $\mathcal{F}_{1}$, it suffices to take two dicritical separatrices $S_{1}$ and $S_{2}$ attached to $D$. Zariski's Theorem gives that they correspond to separatrices $S_{1}^{\prime}$ and $S_{2}^{\prime}$ of $\mathcal{F}_{2}$ which will be attached to a component $D^{\prime}$ produced by the same sequence of blowing-ups as $D$. This argument actually works to any pair of curves passing 
through $D$, thus $D^{\prime}$ will be crossed by infinitely many separatrices and will be dicritical. We will call $D$ and $D^{\prime}$ equivalent dicritical components. This term is also justified by the following:

Proposition 8.1. Let $\mathcal{F}_{1}$ and $\mathcal{F}_{2}$ be topological equivalent foliations. Let $D$ and $D^{\prime}$ be equivalent dicritical components in their desingularizations. Then $D$ and $D^{\prime}$ have the same valence.

Proof. We look at $D$ and $D^{\prime}$ at the very moment they appear in the desingularization process. Denote by $\tilde{\mathcal{F}}_{1}$ and $\tilde{\mathcal{F}}_{2}$ the transforms of $\mathcal{F}_{1}$ and $\mathcal{F}_{2}$ at this step. We first notice that if $p \in D$ is regular for $\tilde{\mathcal{F}}_{1}$ then the Equidesingularization Theorem implies that the leaf $L$ containing $p$ corresponds, by the topological equivalence, to a smooth curve $L^{\prime}$ crossing $D^{\prime}$ transversely at a point $p^{\prime}$. The same occurs for all points near $p$ and this forces $q$ to be a regular point for $\tilde{\mathcal{F}}_{2}$. Let us first prove that $\mathrm{v}(D) \leq \mathrm{v}\left(D^{\prime}\right)$. This is evidently true if $\mathrm{v}(D)=0$. If $\mathrm{v}(D)=1$, since $D^{\prime}$ appears by applying the same sequence of blowing-ups that produce $D$, it arises from a blowing-up at a non-corner point. Thus $\mathrm{v}\left(D^{\prime}\right)$ is 1 at least. The same argument works when $\mathrm{v}(D)=2$, now $D^{\prime}$ also arising from the blowing-up at a non corner point. Let us now suppose that $\mathrm{v}(D)>2$. In the case in which both $D$ and $D^{\prime}$ appear from the blowing-up at a non corner point, in order to proceed towards the desingularization of $\mathcal{F}_{1}$, we will start a sequence of blowing-ups at $\mathrm{v}(D)-1$ different points in $D$, which either correspond to singular points of $\tilde{\mathcal{F}}_{1}$ over $D$ or to points where $\tilde{\mathcal{F}}_{1}$ and $D$ are tangent. In the first case, a convergent separatrix exists by the separatrix Theorem [6]. In the second, the tangent leaf gives rise to a convergent separatrix. Following these separatrices by the topological equivalence, we find $\mathrm{v}(D)-1$ points over $D^{\prime}$ where $\tilde{\mathcal{F}}_{2}$ is not desingularized. We conclude therefore that $\mathrm{v}(D) \leq \mathrm{v}\left(D^{\prime}\right)$. The same argument works when $D$ and $D^{\prime}$ arise from the blowing-up at a corner point. In all cases, we have $\mathrm{v}(D) \leq \mathrm{v}\left(D^{\prime}\right)$. Finally, inverting the roles of $D$ and $D^{\prime}$, we also have $\mathrm{v}\left(D^{\prime}\right) \leq \mathrm{v}(D)$ and this finishes the proof of the proposition.

The main drawback in dealing with topological equivalences is that they do not track formal separatrices as these are not realizable geometric objects. Thus, when considering topological equivalent foliations $\mathcal{F}_{1}$ and $\mathcal{F}_{2}$, it is reasonable to suppose that all separatrices for at least one of the foliations, say $\mathcal{F}_{1}$, are convergent. With this hypothesis, given a balanced equation of separatrices $F_{1}$ for $\mathcal{F}_{1}$, we can choose a balanced equation $F_{2}$ for $\mathcal{F}_{2}$ in such a way that $\Phi_{*}\left(F_{1}\right)_{0} \subset\left(F_{2}\right)_{0}$ and $\Phi_{*}\left(F_{1}\right)_{\infty}=\left(F_{2}\right)_{\infty}$, the inclusion being an equality when all separatrices of $\mathcal{F}_{2}$ are convergent. We can also formulate an analogous statement for balanced equations adapted to sets of separatrices $C$ of $\mathcal{F}_{1}$ and $\Phi_{*} C$ of $\mathcal{F}_{2}$.

Now, our idea is to explore the formula in Theorem B. Let $C_{1}$ be a set of separatrices of $\mathcal{F}_{1}$ - with only convergent separatrices - and $F_{1}$ be a $C_{1}$-adapted balanced equation. Let $F_{2}$ be a balanced set of separatrices for $\mathcal{F}_{2}$ adapted to $C_{2}=\Phi_{*} C_{1}$ as described in the previous paragraph. In Theorem B, we denote

$$
\delta_{0}\left(\mathcal{F}_{i}, C_{i}\right)=-\left[\left(C_{i},\left(F_{i}\right)_{0} \backslash C_{i}\right)_{0}-\left(C_{i},\left(F_{i}\right)_{\infty}\right)_{0}\right], i=1,2 .
$$

With the notations above, the next result is straightforward: 
Proposition 8.2. If all separatrices of $\mathcal{F}_{1}$ are convergent, then

$$
\delta_{0}\left(\mathcal{F}_{1}, C_{1}\right) \geq \delta_{0}\left(\mathcal{F}_{2}, C_{2}\right)
$$

Moreover, equality holds if and only if all separatrices of $\mathcal{F}_{2}$ are convergent.

The next result, a consequence of Theorem B, says in particular that the $\Delta$-index is a topological invariant when both foliations have convergent separatrices.

Proposition 8.3. Suppose that all separatrices of $\mathcal{F}_{1}$ are convergent and let $C_{1}$ be a set of some of its separatrices. Then

$$
\Delta_{p}\left(\mathcal{F}_{1}, C_{1}\right) \leq \Delta_{p}\left(\mathcal{F}_{2}, C_{2}\right),
$$

where $C_{2}=\Phi_{*} C_{1}$. Furthermore, equality holds if and only if all separatrices of $\mathcal{F}_{2}$ are convergent.

Proof. The result follows straight from the topological invariance of the $G S V$ index and from Proposition 8.2.

We close this article by presenting a result on the topological invariance of the algebraic multiplicity. It generalizes at a time the results contained in $[\mathbf{5}]$ and in $[\mathbf{2 2}]$ mentioned in the beginning of the section.

THEOREM F. Suppose that $\mathcal{F}_{1}$ and $\mathcal{F}_{2}$ are topologically equivalent analytic foliations having only convergent separatrices. Then $\mathcal{F}_{1}$ is of second type if and only if $\mathcal{F}_{2}$ is of second type. As a consequence, $\mathcal{F}_{1}$ and $\mathcal{F}_{2}$ have the same algebraic multiplicities.

Proof. We start by remarking that a local foliation always has a separatrix which does not arise as the weak separatrix of a saddle-node. This is the essence of the proof of the Separatrix Theorem in $[6]$. Let $B_{1}$ be such a separatrix for $\mathcal{F}_{1}$ and set $B_{2}=\Phi_{*} B_{1}$. If $\mathcal{F}_{1}$ is of second type then, as a consequence of Proposition $4.5, \Delta_{p}\left(\mathcal{F}_{1}, B_{1}\right)$ does not change along the desingularization of $B_{1}$ and, using Example 4.4, we find $\Delta_{p}\left(\mathcal{F}_{1}, B_{1}\right)=0$. By Proposition 8.3, we also have $\Delta_{p}\left(\mathcal{F}_{2}, B_{2}\right)=0$. Proposition 4.5 then gives $\tau\left(\mathcal{F}_{2}\right)=$ 0 , which means that $\mathcal{F}_{2}$ is a foliation of second type. Now, since a balanced set of separatrices for $\mathcal{F}_{1}$ is taken by $\Phi$ into a balanced set for $\mathcal{F}_{2}$, both sets have the same algebraic multiplicity as a consequence of the Equidesingularization Theorem for curves. Finally, Proposition 3.3 assures that the same will be true for the algebraic multiplicities of $\mathcal{F}_{1}$ and $\mathcal{F}_{2}$.

\section{References}

[1] M. Berthier, R. Meziani and P. Sad, On the classification of nilpotent singularities, Bull. Sci. Math., 123 (1999), 351-370.

[ 2 ] E. Brieskorn and H. Knörrer, Plane algebraic curves, Modern Birkhäuser Classics, Birkhäuser/ Springer Basel AG, Basel, 1986, Translated from the German original by John Stillwell, reprint of the 1986 edition, 2012.

[ 3 ] M. Brunella, Feuilletages holomorphes sur les surfaces complexes compactes, Ann. Sci. École Norm. Sup. (4), 30 (1997), 569-594. 
[4] M. Brunella, Some remarks on indices of holomorphic vector fields, Publ. Mat., 41 (1997), $527-544$.

[5] C. Camacho, A. Lins Neto and P. Sad, Topological invariants and equidesingularization for holomorphic vector fields, J. Differential Geom., 20 (1984), 143-174.

[6 C. Camacho and P. Sad, Invariant varieties through singularities of holomorphic vector fields, Ann. of Math. (2), 115 (1982), 579-595.

[ 7 ] A. Campillo and M. Carnicer, Proximity inequalities and bounds for the degree of invariant curves by foliations of $\mathbf{P}_{\mathbf{C}}^{\mathbf{2}}$, Trans. Amer. Math. Soc., 349 (1997), 2211-2228.

[8] A. Campillo and J. Olivares, Assigned base conditions and geometry of foliations on the projective plane, In: Singularities - Sapporo 1998, Adv. Stud. Pure Math., 29, Math. Soc. Japan, Kinokuniya, Tokyo, 2000, 97-113.

[ 9 ] F. Cano, N. Corral and R. Mol, Local polar invariants for plane singular foliations, to appear in Expo. Math., 2018.

[10] M. Carnicer, The Poincaré problem in the nondicritical case, Ann. of Math. (2), 140 (1994), 289-294.

[11] V. Cavalier and D. Lehmann, Localisation des résidus de Baum-Bott, courbes généralisées et $K$-théorie, I, Feuilletages dans $\mathbb{C}^{2}$, Comment. Math. Helv., 76 (2001), 665-683.

[12] V. Cavalier and D. Lehmann, On the Poincaré inequality for one-dimensional foliations, Compos. Math., 142 (2006), 529-540.

[13] D. Cerveau and A. Lins Neto, Holomorphic foliations in $\mathbf{C P}(2)$ having an invariant algebraic curve, Ann. Inst. Fourier (Grenoble), 41 (1991), 883-903.

[14] D. Cerveau and R. Moussu, Groupes d'automorphismes de (C, 0) et équations différentielles ydy+ $\cdots=0$, Bull. Soc. Math. France, 116 (1988), 459-488.

[15] N. Corral and P. Fernández, Isolated invariant curves of a foliation, Proc. Amer. Math. Soc., 134 (2006), 1125-1132.

[16] E. Esteves and S. Kleiman, Bounds on leaves of one-dimensional foliations, Bull. Braz. Math. Soc. (N.S.), 34 (2003), 145-169.

[17] C. Galindo and F. Monserrat, The Poincaré problem, algebraic integrability and dicritical divisors, J. Differential Equations, 256 (2014), 3614-3633.

[18] Y. Genzmer, Rigidity for dicritical germ of foliation in $\mathbb{C}^{2}$, Int. Math. Res. Not. IMRN, (19):Art. ID rnm072, 2007, 14 pp.

[19] X. Gómez-Mont, J. Seade and A. Verjovsky, The index of a holomorphic flow with an isolated singularity, Math. Ann., 291 (1991), 737-751.

[20] A. Lins Neto, Some examples for the Poincaré and Painlevé problems, Ann. Sci. École Norm. Sup. (4), 35 (2002), 231-266.

[21] J.-F. Mattei and R. Moussu, Holonomie et intégrales premières, Ann. Sci. École Norm. Sup. (4), 13 (1980), 469-523.

[22] J.-F. Mattei and E. Salem, Modules formels locaux de feuilletages holomorphes, arXiv:math/ $0402256,2004$.

[23] M. McQuillan, An introduction to non-commutative Mori theory, In: European Congress of Mathematics, Vol. II (Barcelona, 2000), Progr. Math., 202, Birkhäuser, Basel, 2001, 47-53.

[24] R. Meziani, Classification analytique d'équations différentielles $y d y+\cdots=0$ et espace de modules, Bol. Soc. Brasil. Mat. (N.S.), 27 (1996), 23-53.

[25] R. Meziani and P. Sad, Singularités nilpotentes et intégrales premières, Publ. Mat., 51 (2007), 143-161.

[26] R. Mol, Meromorphic first integrals: some extension results, Tohoku Math. J. (2), 54 (2002), 85-104.

[27] H. Poncaré, Sur l'intégration algébrique des équations différentielles du premier ordre et du premier degré, Rend. Circ. Mat. Palermo, 5 (1891), 161-191.

[28] L. Puchuri, Degree of the first integral of a pencil in $\mathbb{P}^{2}$ defined by Lins Neto, Publ. Mat., 57 (2013), 123-137.

[29] A. Seidenberg, Reduction of singularities of the differential equation $A d y=B d x$, Amer. J. Math., 90 (1968), 248-269. 


\section{Yohann Genzmer}

I. M. T. Université Paul Sabatier

118 Route de Narbonne

31400 Toulouse, France

E-mail: yohann.genzmer@math.univ-toulouse.fr

\section{Rogério MoL}

Departamento de Matemática

Universidade Federal de Minas Gerais

Av. Antônio Carlos, 6627 C.P. 702

30123-970 Belo Horizonte MG, Brazil

E-mail: rmol@ufmg.br 